\title{
THE EXPANSION PROBLEM IN THE THEORY OF ORDINARY LINEAR DIFFERENTIAL SYSTEMS OF THE SECOND ORDER*
}

BY

\author{
RUDOLPH E. LANGER
}

1. Introduction. The system composed of a linear differential equation of order $n$ with boundary conditions applying at the end points of an interval is known to be expressible also as a system of $n$ differential equations of the first order with boundary conditions of corresponding type. In particular, if the original system is of the form

$$
\begin{array}{ll}
u^{(n)}(x)+P_{1}(x, \rho) u^{(n-1)}(x)+\cdots+P_{n}(x, \rho) u(x) & =0, \\
\sum_{l=0}^{n-1}\left\{\alpha_{j l} u^{(l)}(a)+\beta_{j l} u^{(l)}(b)\right\}=0 & (j=1,2, \cdots, n),
\end{array}
$$

with the coefficient $P_{i}(x, \rho)$ a polynomial of degree $i$ in $\rho$, then it may also be written in the specific form

$$
\begin{gathered}
u_{j}^{\prime}(x)=\sum_{l=1}^{n}\left\{p_{j l}(x) \rho+q_{j l}(x)\right\} u_{l}(x), \\
\sum_{l=1}^{n}\left\{\sigma_{j l} u_{l}(a)+\tau_{j l} u_{l}(b)\right\}=0 \quad(j=1,2, \cdots, n) . \dagger
\end{gathered}
$$

Moreover, if in the original system (1) the coefficients of the boundary conditions are polynomials in the parameter, the same is also true when the system is written in the form (2).

The systems (1) and (2) are both adapted to a consideration of the associated boundary problems and both of these problems have been discussed under hypotheses of considerable generality. $\ddagger$ An essential restriction, however, which has been imposed in all but a few investigations dealing with sys-

\footnotetext{
* Presented to the Society, September 7, 1928, and August 29, 1929; received by the editors in June, 1928, and May, 1929.

$\dagger$ Wilder, C. E., these Transactions, vol. 29 (1927), p. 497.

$\ddagger$ Cf. in the case of system (1), Tamarkin 1. Some General Problems of the Theory of Ordinary Linear Differential Equations, etc., Petrograd, 1917 (in Russian), 2. Mathematische Zeitschrift, vol. 27 (1927), p. 1; and in the case of system (2), Birkhoff and Langer, Proceedings of the American Academy of Arts and Sciences, vol. 58 (1923), p. 51.
} 
tems of specialized types, is that certain so-called conditions of "regularity" be fulfilled.*

The present paper is a discussion of the systems (1) and (2) of the second order, and a first purpose of the paper is the consideration of the boundary problems when certain of the major hypotheses of regularity are omitted. This weakening of the restrictions imposed in general involves a sacrifice of the convergence of the series expansions for arbitrary functions in terms of the solutions of the differential system. It is shown, however, that in a wide class of cases in which these expansions are non-convergent, they are nevertheless summable by suitable means.

Beyond this extension of the theories to irregular cases a second purpose of the paper is to equalize the extent of development of the theories of the two systems. While, on the one hand, the system (2) includes the system (1) and is somewhat more general, the investigations concerned with it have hitherto been confined to the case in which the coefficients of the boundary conditions are free from the parameter. $\dagger$ In the present discussion the coefficients are taken to be polynomials and in this respect the development of the theory of system (2) is brought to a par with that of the system (1). $f$ In the matter of the expansibility of arbitrary functions in series of characteristic solutions, on the other hand, the theory of system (2) includes theorems asserting that $n$ such functions may be simultaneously expanded with a single determination of coefficients, whereas in this respect the theory of system (1) has not hitherto been extended beyond the theorems on the expansibility of a single function. The simultaneous expansions associated with system (1) are included in the following considerations.

Representation of the differential system in matrix form is largely but not exclusively used as the most convenient scheme of notation. To obviate confusion between matrix and scalar quantities, however, the former are exclusively designated by script capitals. In the case of a matrix in which both columns are the same the designating letter is modified by a succeeding dot, while in the case of a matrix in which both rows are the same a preceding dot is used. Such matrices will be referred to as vectors. As a general rule

* The irregular system of type (1) and second order in which the differential equation is of a specialized form and the boundary conditions are free from the parameter has been considered by Stone, these Transactions, vol. 29 (1927), p. 23. Certain special irregular systems of the third order have been considered by Hopkins, these Transactions, vol. 20 (1919), p. 245, and by Ward, Annals of Mathematics, (2), vol. 26 (1924), p. 21, and these Transactions, vol. 29 (1927), p. 716.

$\dagger$ Cf. Birkhoff and Langer, loc. cit.

$\ddagger$ Tamarkin 2, loc. cit. 
the notation in so far as matrices are concerned is that used by Birkhoff and the author in the paper cited.*

2. The differential system of type (1). The explicit form of the system of type (1) to be considered is

$$
\begin{gathered}
u^{\prime \prime}(\xi)+\left\{p_{11}(\xi) \rho+p_{10}(\xi)\right\} u^{\prime}(\xi)+\left\{p_{22}(\xi) \rho^{2}+p_{21}(\xi) \rho+p_{20}(\xi)\right\} u(\xi)=0, \\
\alpha_{j 1}(\rho) u^{\prime}(a)+\alpha_{j 0}(\rho) u(a)+\beta_{j 1}(\rho) u^{\prime}(b)+\beta_{j 0}(\rho) u(b)=0, j=1,2,
\end{gathered}
$$

in which the $\alpha_{j l}, \beta_{j l}$ are any polynomials in $\rho$. The coefficients of the system may be real or complex. To obviate discussions not essential to the primary purpose of the paper, we shall suppose them to have derivatives of sufficiently high orders on the interval $(a, b)$. As explicit hypotheses on the system (3), however, we shall assume as is usual $\dagger$ that on $(a, b)$ the $\operatorname{roots} \theta_{1}(\xi), \theta_{2}(\xi)$, of the equation

$$
\theta^{2}+p_{11}(\xi) \theta+p_{22}(\xi)=0
$$

are distinct, non-vanishing, and of constant argument, i.e.,

$$
\begin{gathered}
\theta_{1}(\xi) \neq \theta_{2}(\xi), \\
\theta_{j}(\xi)=\left|\theta_{j}(\xi)\right| e^{i c_{j}} \neq 0, j=1,2 .
\end{gathered}
$$

If we suppose, as we may, that the arguments $c_{j}$ are determined so that

$$
\left|c_{1}-c_{2}\right| \leqq \pi
$$

the change of variables and parameter

$$
u=\psi(\xi) y, \quad x=(b-a)^{-1}(\xi-a), \quad \rho=\lambda e^{-i\left(c_{1}+c_{2}\right) / 2},
$$

with a suitable determination of the function $\psi(\xi)$, reduces the interval $(a, b)$ to $(0,1)$ and the system $(3)$ to the form

(a) $y^{\prime \prime}(x)-\left\{\lambda\left(r_{1}(x)+r_{2}(x)\right)+q_{1}(x)\right\} y^{\prime}(x)+\left\{\lambda^{2} r_{1}(x) r_{2}(x)\right.$

$$
\left.+\lambda\left(r_{1}(x) q_{1}(x)-r_{1}^{\prime}(x)\right)+q_{2}(x)\right\} y(x)=0,
$$

(b) $\omega_{j 1}(\lambda) y^{\prime}(0)+\omega_{j 2}(\lambda) y(0)+\omega_{j 3}(\lambda) y^{\prime}(1)+\omega_{j 4}(\lambda) y(1)=0, \quad j=1,2$.

In this form, which we designate as the normal form, the coefficients $\omega_{j l}(\lambda)$ are again polynomials, and if we write

we have

$$
r_{j}(x)=\nu_{j}\left|r_{j}(x)\right|
$$

* The reader is referred to that paper for a brief introductory exposition of the differential system in matrix form.

† Cf. Tamarkin 2, loc. cit., pp. 3 and 21; Birkhoff and Langer, loc. cit., pp. 72 and 109. Bliss, however, has considered the differential system with a set of conditions under which this hypothesis may not be fulfilled; these Transactions, vol. 28 (1926), p. 561. 
(a)

$$
\left|r_{j}(x)\right|>0, j=1,2 \text {; }
$$

$$
\begin{aligned}
\nu_{1}=\nu_{2}^{-1} & =e^{i \alpha}, & 0 \leqq \alpha \leqq \pi / 2 \quad(\alpha \text { constant }) ; \\
& r_{1}(x) \neq r_{2}(x) . &
\end{aligned}
$$

The system (5) may now be expressed in the form of the differential system (2) by setting $y=y_{1}$, and writing

$$
\begin{aligned}
& y_{1}^{\prime}=\lambda r_{1}(x) y_{1}+e^{\int_{0}^{x} a_{1} d x} y_{2}, \\
& y_{2}^{\prime}=\lambda r_{2}(x) y_{2}-q_{2}(x) e^{-\int_{0}^{x} q_{1} d x} y_{1} .
\end{aligned}
$$

3. The matrix form of system (2). In the notation of matrices the system (2) takes the form

$$
\begin{aligned}
& U^{\prime}(\xi)=\{P(\xi) \rho+2(\xi)\} U(\xi), \\
& \mathscr{W}_{a} U(a)+\mathscr{W}_{b} U(b)=\mathcal{O},
\end{aligned}
$$

the elements of any column of a matrix solution $U(\xi)$ constituting a solution of system (2). This system we shall consider for the case in which the elements of the matrices $\mathscr{W}_{a}$ and $\mathscr{W}_{b}$ are any polynomials in $\rho$, and we shall suppose as in $\$ 2$ that the coefficients involved have derivatives of such orders as may be required. With the hypothesis, which we shall now make, that the roots $\theta_{1}(\xi), \theta_{2}(\xi)$, of the determinant equation

$$
\left|\begin{array}{cc}
p_{11}(\xi)-\theta & p_{12}(\xi) \\
p_{21}(\xi) & p_{22}(\xi)-\theta
\end{array}\right|=0,
$$

satisfy the conditions (4), we may without loss of generality suppose that in system $(8), p_{j l}(\xi)=\delta_{j l} \theta_{j}(\xi),{ }^{*}$ where $\delta_{j j}=1, \delta_{j l}=0, j \neq l$.

The functions $\psi_{1}(\xi), \psi_{2}(\xi)$ may now be determined so that the change of variables and parameter

$$
U(\xi)=\left(\delta_{i} \psi_{j}(\xi)\right) \Upsilon(\xi), \quad x=(b-a)^{-1}(\xi-a), \quad \rho=\lambda^{-i\left(c_{1}+c_{2}\right) / 2},
$$

reduces the system (8) to the normal form

$$
\begin{aligned}
\Upsilon^{\prime}(x)-\{R(x) \lambda+\mathbb{B}(x)\} \Upsilon(x) & =\mathcal{O}, \\
\mathscr{W}_{12}(\lambda) \Upsilon(0)+\mathscr{W}_{34}(\lambda) \Upsilon(1) & =\mathcal{O} .
\end{aligned}
$$

In this form the elements of the matrices $\mathscr{W}_{12}$ and $\mathscr{W}_{43}$ are again polynomials, while

* Cf. Birkhoff and Langer, loc. cit., p. 72. Throughout this paper the symbol $\delta_{j l}$ will be used exclusively to designate the "Kronecker delta" as in the text. 


$$
R(x)=\left|\begin{array}{cc}
r_{1}(x) & 0 \\
0 & r_{2}(x)
\end{array}\right|, \quad B(x)=\left|\begin{array}{cc}
0 & b_{1}(x) \\
b_{2}(x) & 0
\end{array}\right|,
$$

and the functions $r_{1}(x), r_{2}(x)$ satisfy the conditions (6).

We observe now that the pair of equations (7) are precisely in the form of the component equations of the vector system corresponding to (9), i.e., with

$$
\begin{array}{r}
\Upsilon^{\prime}(x) \cdot-\{R(x) \lambda+\mathscr{B}(x)\} \Upsilon(x) \cdot=\mathcal{O} \\
\mathscr{W}_{12}(\lambda) \Upsilon(0) \cdot+\mathfrak{W}_{34}(\lambda) \Upsilon(1) \cdot=\mathcal{O}
\end{array}
$$

with

$$
b_{1}(x)=e^{\int_{0}^{x} a_{1}(x) d x}, \quad b_{2}(x)=-q_{2}(x) e^{-\int_{0}^{x} q_{1}(x) d x} .
$$

Hence the equation (5a) is equivalent to one of form (10a) in which the element $b_{1}(x)$ vanishes nowhere in the interval. Inasmuch as this property is invariant under a change of variables the greater generality of system (10) without restriction of $b_{1}(x)$ is evident. Any conclusions to be derived for the system (10) will be expressible in terms of the system (5) by means of the relations

$$
\begin{array}{ll}
y_{1}(x)=y(x), & y(x)=y_{1}(x), \\
y_{2}(x)=\left\{y^{\prime}(x)-\lambda r_{1}(x) y(x)\right\} e^{-\int_{0}^{x} q_{1} d x} ; y^{\prime}(x)=\lambda r_{1}(x) y_{1}(x)+e \int_{0}^{x} q_{1} d x y_{2}(x) .
\end{array}
$$

4. The asymptotic solutions. The asymptotic forms of certain solutions of the matrix equation (9a) and its adjoint equation

$$
Z^{\prime}(x)+Z(x)\{R(x) \lambda+B(x)\}=\mathcal{O},
$$

are known* when $\lambda$ is confined to any region of the $\lambda$ plane in which

$$
\operatorname{Re}\left(\lambda r_{1}(x)-\lambda r_{2}(x)\right) \dagger
$$

either remains greater than or less than some constant for all $x$ of the interval $(0,1)$. To insure the availability of these asymptotic forms over the entire $\lambda$ plane we make the usual hypothesis $\ddagger$ that

$$
\arg \left\{r_{1}(x)-r_{2}(x)\right\}=A \quad \text { (a constant). }
$$

This imposes no restriction on the functions $r_{j}(x)$ beyond those already involved in (6), when the argument $\alpha$ of (6b) is either 0 or $\pi / 2$, for irrespective of the functions $r_{j}(x)$ the quantity (14) has in the former of these cases the

* Cf. Birkhoff and Langer, loc. cit., p. 87.

† The symbol $\operatorname{Re}(\mu)$ will be used to designate "the real part of $\mu . "$

$\ddagger$ Birkhoff and Langer, loc. cit., p. 83; Tamarkin 2, loc. cit., p. 21. 
constant value 0 or $\pi$, as is evident from (6b) and (6c), while in the latter case it has the constant value $\pi / 2$. When $0<\alpha<\pi / 2$, however, the restriction imposed by the hypothesis (14) is clearly that the ratio $r_{1}(x) / r_{2}(x)$ be a constant.

We adopt now the familiar notation $[a(x)]$ to designate an expression of the form

$$
[a(x)]=a(x)+\frac{a_{1}(x)}{\lambda}+\cdots+\frac{a_{l}(x)}{\lambda^{l}}+\frac{E(x, \lambda)}{\lambda^{l+1}},
$$

where $l$ is any desired integer, and $E(x, \lambda)$ denotes a function which is analytic in $\lambda$ and bounded for $|\lambda|$ sufficiently large. If we abbreviate then, by setting

$$
R_{j}(x, t)=\int_{t}^{x} r_{j}(x) d x, \quad R_{j}(x)=R_{j}(x, 0), \quad R_{j}=R_{j}(1,0),
$$

the asymptotic solutions for the equations (9a) and (13), when $\lambda$ remains in any half-plane bounded by a line parallel to the ray

$$
\arg \lambda=\pi / 2-A,
$$

are respectively as follows:

$$
\Upsilon(x)=P(x, \lambda) \mathcal{E}(x, \lambda),
$$

where

$$
\begin{aligned}
e_{i j}(x, \lambda) & =\delta_{i j} e^{\lambda R_{j}(x)}, \\
p_{i j}(x, \lambda) & =\lambda^{\delta_{i j}-1}\left[p_{i j}(x)\right], p_{i i}(x) \equiv 1 ;
\end{aligned}
$$

and

$$
\begin{aligned}
Z(x) & =\mathcal{E}^{-1}(x, \lambda) \mathcal{Q}(x, \lambda), \\
q_{i j}(x, \lambda) & =\lambda^{\delta_{i j}-1}\left[q_{i j}(x)\right], \quad q_{i i}(x) \equiv 1 .
\end{aligned}
$$

Each column of the matrix (16a) is a solution of the vector equation (10a) and each row of the matrix (17a) is a solution of the vector equation

$$
\cdot Z^{\prime}(x)+\cdot Z(x)\{R(x) \lambda+\mathbb{B}(x)\}=\mathcal{O} .
$$

5. The boundary conditions. The adjoint system. The boundary conditions which together with the equation (18) yield the vector system adjoint to (11) are of the form

$$
\cdot z(0) \cup+\cdot z(1) \bar{v}=\mathcal{O}
$$


the matrices $v$ and $\bar{v}$ being required to satisfy the conditions (i), that the rectangular array of their elements

$$
\left\|v_{i j}, \bar{v}_{i j}\right\| \text { be of rank } 2,
$$

and (ii), that

$$
\mathfrak{W}_{12} v-\mathfrak{W}_{34} \bar{v}=\mathcal{O}^{*}
$$

To obtain these boundary conditions in terms of the elements of the matrices in (10b) we proceed as follows.

Let $W_{j l}(\lambda)$ be defined as the matrix whose elements are those of the $j$ th and $l$ th columns of the array

$$
\left\|\begin{array}{llll}
w_{11}(\lambda) & w_{12}(\lambda) & w_{13}(\lambda) & w_{14}(\lambda) \\
w_{21}(\lambda) & w_{22}(\lambda) & w_{23}(\lambda) & w_{24}(\lambda)
\end{array}\right\| .
$$

From consistency of this definition with the notation of equation (10b), it follows that the elements of (22) are those of the matrices $\mathscr{W}_{12}$ and $\mathfrak{W}_{34}$, and are, therefore, polynomials in $\lambda$. Moreover, our hypotheses must include the assumption that for all values of $\lambda$ the array (22) is of rank two. We now designate by $\Im_{h l}$, the matrix whose elements are zero excepting that in the $h$ th row and $l$ th column which is unity, i.e.,

$$
J_{h l}=\left(\delta_{i h} \delta_{l_{j}}\right) .
$$

Then it is readily verified that we may write

$$
\begin{aligned}
& \mathfrak{W}_{12}=\mathscr{W}_{h, 2+l} \Im_{1 h}+W_{m, 2+n} \Im_{1 m}, \\
& \mathfrak{W}_{34}=\mathscr{W}_{h, 2+l} \Im_{2 l}+W_{m, 2+n} J_{2 n} ; \quad h, l=1,2 ; \quad h+m=l+n=3,
\end{aligned}
$$

with any choice of the subscripts subject to the restrictions indicated. If $\Upsilon_{h l} \cdot$ and $\cdot Z_{h l}$ are then defined by the relations

$$
\begin{aligned}
& \Upsilon_{12} \cdot=\Upsilon(0) \cdot, \quad \Upsilon_{34} \cdot=\Upsilon(1) \cdot, \\
& \Upsilon_{h l}=J_{1 h} \Upsilon(0) \cdot+J_{2, l-2} \Upsilon(1) \cdot, \text { for } h=1,2 ; l=3,4 ;
\end{aligned}
$$

and

$$
\begin{aligned}
& \cdot Z_{12}=-\cdot Z(0), \quad \cdot Z_{34}=\cdot Z(1), \\
& \cdot Z_{h l}=-\cdot Z(0) J_{h 1}+\cdot Z(1) J_{l-2,2}, \quad \text { for } \quad h=1,2 ; l=3,4 ;
\end{aligned}
$$

it follows that

$$
W_{12} \Upsilon(0) \cdot+W_{34} \Upsilon(1) \cdot \equiv W_{h l} \Upsilon_{h l} \cdot+W_{m n} \Upsilon_{m n} \cdot,
$$

and furthermore that

* Bliss, loc. cit., p. 562. 


$$
\left.\cdot Z(x) \Upsilon(x) \cdot\right|_{0} ^{1}=\cdot Z_{h l} \Upsilon_{h l} \cdot+\cdot Z_{m n} \Upsilon_{m n} \cdot
$$

In these relations the subscripts may be chosen as any permutation of the numbers $1,2,3,4$, subject to $h<l$ and $m<n$.

Now for any value of $\lambda$ the subscripts $h, l$ may be so chosen that $\mathscr{W}_{h l}^{-1}(\lambda)$ exists. Then condition (10b) is found with the use of (26) to reduce the matrix (27) to the zero matrix if the vector $\cdot Z(x)$ satisfies the condition

$$
\cdot Z_{m n}-\cdot Z_{h l} W_{h l}^{-1} \mathscr{W}_{m n}=\mathcal{O} \text {. }
$$

This relation expressed in the form (19), as it may be by the use of (25), is easily shown to satisfy the conditions (20) and (21). Hence we may write the system (10) and its adjoint system in the corresponding forms

$$
\text { (a) } \begin{aligned}
\Upsilon^{\prime}(x) \cdot-\{R(x) \lambda+\mathscr{B}(x)\} \Upsilon(x) \cdot & =\mathcal{O}, \\
\text { (b) } & \Upsilon_{h l} \cdot+\mathscr{W}_{h l}^{-1}(\lambda) \mathscr{W}_{m n}(\lambda) \Upsilon_{m n} \cdot=\mathcal{O}, \quad h<l ; m<n ;
\end{aligned}
$$

and

(a) $\cdot Z^{\prime}(x)+\cdot Z(x)\{R(x) \lambda+\mathscr{B}(x)\}=\mathcal{O}$,

$$
\text { (b) } \quad \cdot Z_{m n}-\cdot Z_{h l} \mathscr{W}_{h l}^{-1}(\lambda) \mathscr{W}_{m n}(\lambda)=\mathcal{O}, \quad h<l ; m<n \text {. }
$$

6. The characteristic values. The characteristic equation whose roots are the values of $\lambda$ for which the system (10) admits of a solution other than the trivial solution $\Upsilon(x) \cdot \equiv \mathcal{O}$, is obtained by equating to zero the determinant of the matrix on the left of equation (9b).* With the form (16a) substituted for $\Upsilon(x)$, this equation may be written

$$
D(\lambda)=0 \text {, }
$$

where

$$
D(\lambda) \equiv\left|\mathscr{W}_{12}(\lambda) \mathcal{P}(0, \lambda)+\mathscr{W}_{34}(\lambda) \mathcal{P}(1, \lambda) \mathcal{E}(1, \lambda)\right| .
$$

If the values given by (16b) and (16c) are substituted in this expression for $D(\lambda)$, the latter becomes explicitly

$$
D(\lambda) \equiv\left|\begin{array}{ll}
d_{11}(\lambda)+d_{13}(\lambda) e^{\lambda R_{1}} & d_{12}(\lambda)+d_{14}(\lambda) e^{\lambda R_{2}} \\
d_{21}(\lambda)+d_{23}(\lambda) e^{\lambda R_{1}} & d_{22}(\lambda)+d_{24}(\lambda) e^{\lambda R_{2}}
\end{array}\right|,
$$

where we have abbreviated by setting

$$
\begin{aligned}
d_{i j}(\lambda) & =w_{i 1}(\lambda) p_{1 j}(0, \lambda)+w_{i 2}(\lambda) p_{2 j}(0, \lambda), \\
d_{i, j+2}(\lambda) & =w_{i 3}(\lambda) p_{1 j}(1, \lambda)+w_{i 4}(\lambda) p_{2 j}(1, \lambda), \quad i, j=1,2 .
\end{aligned}
$$

* Birkhoff and Langer, loc. cit., p. 87. 
For convenience in the discussion of equation (30), as well as for later reference, we define the determinants $D_{r}(\lambda)$ by the formulas

$$
\begin{aligned}
& D_{0}(\lambda)=\left|d_{i 1}, d_{i 2}\right|, \quad D_{1}(\lambda)=\left|d_{i 3}, d_{i 2}\right|, \quad D_{2}(\lambda)=\left|d_{i 1}, d_{i 4}\right|, \\
& D_{3}(\lambda)=\left|d_{i 3}, d_{i 4}\right|, D_{4}(\lambda)=\left|d_{i 2}, d_{i 4}\right|, D_{5}(\lambda)=\left|d_{i 3}, d_{i 1}\right| \text {. }
\end{aligned}
$$

In expanded form the expression (32) is, then,

$$
D(\lambda) \equiv D_{0}(\lambda)+D_{1}(\lambda) e^{\lambda R_{1}}+D_{2}(\lambda) e^{\lambda R_{2}}+D_{3}(\lambda) e^{\lambda\left(R_{1}+R_{3}\right\}},
$$

and in this the determinant $D_{r}(\lambda)$, in virtue of (33) and (16c), either vanishes identically or else is of the form

$$
D_{r}(\lambda)=\lambda^{\kappa_{r}}\left[\alpha_{r}\right]
$$

with $\alpha_{r} \neq 0$ and $\kappa_{r}$ an integer.

The conditions of "regularity" of the boundary problem to which reference has already been made, and which are usually imposed,* may be summarized here briefly as follows: If in (6b) $\alpha=0$, then $D_{0} D_{3} \neq 0$, and $\kappa_{0}=\kappa_{3} \geqq \kappa_{1}, \kappa_{2}, \kappa_{4}, \kappa_{5} ;$ if $\alpha=\pi / 2$ then $D_{1} D_{2} \neq \equiv 0$, and $\kappa_{1}=\kappa_{2} \geqq \kappa_{0}, \kappa_{3}, \kappa_{4}, \kappa_{5}$; and if $0<\alpha<\pi / 2$ then $D_{r} \not \equiv 0, r=0,1,2,3$, and $\kappa_{0}=\kappa_{1}=\kappa_{2}=\kappa_{3} \geqq \kappa_{4}, \kappa_{5}$. In the present discussion we shall omit entirely these restrictions on the values of the integers $\kappa_{r}$.

The transcendental equation $D(\lambda)=0$, under variously restrictive hypotheses, has been discussed by Wilder, Tamarkin, Pólya and Schwengler. A discussion of the equation under hypotheses sufficiently general to include those of the present case has been given by the author, $\dagger$ and we shall therefore be content here merely to summarize the salient facts. If each coefficient $D_{r}(\lambda)$ in (35) is identically zero the characteristic equation (30) imposes no restriction, and the system (10) accordingly admits of a solution for every value of $\lambda$. On the other hand, if one and only one coefficient $D_{r}(\lambda)$ in (35) fails to vanish identically the characteristic equation can have no roots outside of a sufficiently large circle in the $\lambda$ plane $\ddagger$. In these cases there is no expansion problem. We shall not consider them further, but shall suppose in proceeding that at least two terms of $D(\lambda)$ actually occur.

* Tamarkin 2, loc. cit., pp. 23, 25; Birkhoff and Langer, loc. cit., p. 89.

$\dagger$ In the present issue of the Transactions, pp. 837-844.

$\ddagger$ Examples of these cases are easily given. Thus, if in equation $(9 a) r_{1}(x)$ and $r_{2}(x)$ are any distinct constants, and $b_{1}$ is a constant and $b_{2} \equiv 0$, we have as an actual solution of the equation the matrix

$$
\Upsilon(x)=\left(\begin{array}{cc}
e^{\lambda r_{1} x} & \frac{\beta}{\lambda} e^{\lambda r_{2} x} \\
0 & e^{\lambda r_{2} x}
\end{array}\right), \text { where } \beta=b_{1} /\left(r_{2}-r_{1}\right) .
$$

Then with the boundary condition

$$
\mathscr{W}_{12}(\lambda)=\left(\begin{array}{rr}
\lambda & -\beta \\
0 & 0
\end{array}\right), \mathscr{W}_{34}(\lambda)=\left(\begin{array}{rr}
0 & 0 \\
-\lambda & \beta
\end{array}\right),
$$


In the case in which the constant $\alpha$ in (6b) is zero, the characteristic values lie in strips of asymptotically constant width which for $|\lambda|$ sufficiently large lie within arbitrarily small sectors including the positive and negative axes of imaginaries. The bounding curves of the strips, moreover, approach parallelism with these axes, and if $l$ is any constant, then the number $N_{t}$ of characteristic values lying between the circles $|\lambda|=\Lambda$, and $|\lambda|=\Lambda+l$, satisfies, when $\Lambda$ is sufficiently large, a relation of the form

$$
a l-b \leqq N_{l} \leqq a l+b,
$$

$a$ and $b$ being specific constants. The case in which $\alpha=\pi / 2$ is similar to that in which $\alpha=0$, with the exception that the strips containing the characteristic values then lie in small sectors about the axes of reals and approach parallelism with these axes.

Finally in the case $0<\alpha<\pi / 2$ the characteristic values lie asymptotically at regular intervals along specific curves which approach parallelism with certain rays in the $\lambda$ plane determined by the value $\alpha$. If in the expression (35) no term vanishes identically, these rays are given by the formula

$$
\arg \lambda= \pm \pi / 2 \pm \alpha,
$$

and there is just one curve marking the asymptotic location of characteristic values in any small sector enclosing one of these rays. If, on the other hand, the terms in (35) are not all different from zero, some of the rays (37) will have no corresponding curves of characteristic values and certain other rays in the plane may have that property. Inasmuch as we shall not consider the expansion problem in these latter cases, we shall go no further into details.

It is convenient for the considerations which follow to assign subscripts to the characteristic values in the order of their numerical magnitude, i.e., so that

$$
\left|\lambda_{s}\right| \leqq\left|\lambda_{s+1}\right|
$$

As is customary, we shall designate the solutions of the systems (10) and (29). corresponding to the characteristic value $\lambda_{s}$, by $\Upsilon^{*}(x) \cdot$ and $\cdot Z^{*}(x)$ respectively.

system (10) is found to admit of the solution

$$
\Upsilon(x)=\left(\begin{array}{c}
\beta e^{\lambda r_{2} x} \\
\lambda e^{\lambda_{2} x}
\end{array}\right) \cdot
$$

for every value of $\lambda$. On the other hand with the value $\mathscr{W}_{12}(\lambda)$ above and

the characteristic equation is

$$
\mathscr{W}_{34}(\lambda)=\left(\begin{array}{cc}
0 & 1 \\
-\lambda & \beta
\end{array}\right) \text {, }
$$

and hence is satisfied only for $\lambda=0$.

$$
\lambda e^{\lambda\left(r_{1}+r_{2}\right\}}=0,
$$


7. The contours $C_{n}$. It is clear from the discussion of the preceding section that regardless of the value of $\alpha$ the number of characteristic values which lie in any given annular region with center at $\lambda=0$, width $l$, and inner radius of sufficient magnitude, is subject to a relation of the type (36). Hence it is seen directly that it is always possible to construct in the $\lambda$ plane an infinite sequence of closed contours $C_{n}, n=n_{1}, n_{2}, \cdots$, having the following properties:

(i) Every point of any contour $C_{n}$ lies at a distance greater than a definite positive constant $\delta$ from any characteristic value;

(ii) The contour $C_{n}$ cuts the rays (37) and the axes of reals and imaginaries at the distance $\Lambda_{n}$ from the origin, where $\Lambda_{n} \rightarrow \infty$ as $n \rightarrow \infty$ over the values $n_{1}, n_{2}, \cdots$;

(iii) The contour $C_{n}$ contains in its interior just $n$ characteristic values;

(iv) The number of characteristic values between two consecutive contours is bounded, i.e., $n_{r+1}-n_{r} \leqq N$, for all $r$.

These contours $C_{n}$ are conveniently thought of as circles with centers at $\lambda=0$ and respective radii $\Lambda_{n}$, and to avoid unessential complications we shall in the subsequent discussions consider them in that way.

A further fact which we note here for future reference is that the quotient of $D(\lambda)$ by any one of its terms as given in (35) is bounded uniformly from zero provided, firstly, that $\lambda$ remains uniformly away from the characteristic values and, secondly, that $|\lambda|$ is sufficiently large.* Since by property (i) above this condition is satisfied when $\lambda$ is confined to the contours described, it follows that any quotient obtained by dividing a term of (35) by $D(\lambda)$ remains uniformly bounded on the contours $C_{n}$.

8. The formal expansion of an arbitrary vector. Let $\mathcal{F}(x)$. be a vector chosen arbitrarily with integrable elements $f_{1}(x)$ and $f_{2}(x)$. We consider formally the possibility of developing such a vector in a series of characteristic vectors $\Upsilon^{s}(x) \cdot$, and seek to determine the coefficients $c_{s}$ in the relation

$$
\mathcal{F}(x) \cdot \approx \sum_{s=1}^{\infty} c_{s} \Upsilon^{s}(x) \cdot .
$$

The symbol $\approx$ is used here to emphasize the fact that the equality is implied in a formal sense only. The following deductions are to be purely heuristic and hence the assumptions to be made in the course of the reasoning are not to be considered as essential. In a later section the rigorous consideration of the results to be obtained will be made.

* Langer, loc. cit. 
If we abbreviate by designating the left-hand members of equations (28a) and (29a) by $\mathcal{L}(\Upsilon, \lambda) \cdot$ and $\cdot \mathscr{H}(\mathcal{Z}, \lambda)$, respectively, i.e.,

$$
\begin{aligned}
& \mathcal{L}(\Upsilon, \lambda) \cdot \equiv \Upsilon^{\prime}(x) \cdot-\{R(x) \lambda+\mathbb{B}(x)\} \Upsilon(x) \cdot, \\
& \cdot \mathscr{H}(Z, \lambda) \equiv \cdot \mathcal{Z}^{\prime}(x)+\cdot \mathcal{Z}(x)\{R(x) \lambda+\mathcal{B}(x)\},
\end{aligned}
$$

the relation

$$
\cdot Z^{*}(x) \mathcal{L}\left(\Upsilon^{r}, \lambda_{r}\right) \cdot+\cdot \mathscr{H}\left(Z^{s}, \lambda_{s}\right) \Upsilon^{r}(x) \cdot=\mathcal{O}
$$

is an identity in the values $r$ and $s$. Upon integration we obtain from it the equation

$$
\left(\lambda_{s}-\lambda_{r}\right) \int_{0}^{1} \cdot Z^{s} R \Upsilon^{r} \cdot d x+\left.\cdot Z^{s}(x) \Upsilon^{r}(x) \cdot\right|_{0} ^{1}=\mathcal{O}
$$

If $W_{h l}(\lambda)$ designates, now, the determinant of the corresponding matrix $\mathscr{W}_{h l}(\lambda)$, this determinant is a polynomial in $\lambda$, and if there are characteristic values for which the determinant vanishes they can be only finite in number. With the possible exception of a limited number of values of $r$ and $s$, then, the final matrix in the left-hand member of (39) can be written, in virtue of (27), (28b) and (29b), in the form

$$
\left.\cdot Z^{s} \Upsilon^{r} \cdot\right|_{0} ^{1} \equiv \cdot Z_{h l}^{s}\left\{W_{h l}^{-1}(\lambda) W_{m n}(\lambda)\right\}_{\lambda=\lambda_{r}}^{\lambda=\lambda_{s}} \Upsilon_{m n}^{r} \cdot
$$

Moreover, if the subscripts $h, l$ are chosen so that the degree of the determinant $W_{h l}(\lambda)$ is at least as high as that of any other determinant formed from the columns of the array (22), we may write

$$
\frac{1}{\lambda_{s}-\lambda_{r}}\left\{W_{h l}^{-1}(\lambda) W_{m n}(\lambda)\right\}_{\lambda_{2=\lambda_{r}}}^{\lambda-\lambda_{s}}=\sum_{k=0}^{K} \frac{\lambda_{s}^{k}}{W_{h l}\left(\lambda_{s}\right)} 2^{k}\left(\lambda_{r}\right),
$$

where the elements of the matrix $2^{k}(\lambda)$ are rational in $\lambda$, and $K+1$ is at most as great as the degree of $W_{h l}(\lambda)$. If $\lambda_{r} \neq \lambda_{s}$ it follows from this that relation (39) may be expressed in the form

$$
\int_{0}^{1} \cdot Z^{*} R \Upsilon^{r} \cdot d x+\cdot Z_{h l}^{s} \sum_{k=0}^{K} \frac{\lambda_{s}{ }^{k}}{W_{h l}\left(\lambda_{s}\right)} 2^{k}\left(\lambda_{r}\right) \Upsilon_{m n}^{r} \cdot=\mathcal{O}
$$

If we suppose in proceeding that the characteristic values are all simple and distinct from the zeros of $W_{h l}(\lambda)$, the relation (41) is valid except possibly for $s=r$. Hence on multiplying the equation (41) by $c_{r}$, the general coefficient in (38), and summing with respect to $r$ we obtain formally with the use of (38) the relation 


$$
\begin{aligned}
\int_{0}^{1} \cdot Z \cdot R \mathcal{F} \cdot d x & +\cdot Z_{h l}^{s} K\left(\lambda_{s}\right) \cdot \\
& =c_{s}\left\{\int_{0}^{1} \cdot Z^{*} R Y^{s} \cdot d x+\cdot Z_{h l}^{s} \sum_{k=0}^{K} \frac{\lambda_{s}{ }^{k}}{W_{h l}\left(\lambda_{s}\right)} 2^{k}\left(\lambda_{s}\right) Y_{m n}^{s} \cdot\right\} .
\end{aligned}
$$

In this we have abbreviated by setting

$$
\sum_{r=1}^{\infty} c_{r} 2^{k}\left(\lambda_{r}\right) \Upsilon_{m n}^{r} \cdot=\mathfrak{F}^{k} . \quad(k=0,1,2, \cdots, K),
$$

and

$$
\sum_{k=0}^{K} \frac{\lambda_{s}{ }^{k}}{W_{h l}\left(\lambda_{s}\right)} \mathcal{K}^{k} .=K\left(\lambda_{s}\right) \cdot
$$

The relation (42) involves only matrices in which all elements are the same. Hence if we suppose the matrix on the right to be different from $\mathcal{O}$, the equality of the general element on the left with the general element on the right determines the constant $c_{8}$.

It is true that the matrix $K\left(\lambda_{s}\right)$. apparently involves the constants $c_{r}$ through the relations (44) and (45), and that therefore the determination of $c_{s}$ by (42) is elusive. We may formally obviate the difficulty in this point by supposing that the constants $c_{r}$ can be chosen to yield not merely relation (38) but also and simultaneously the relations (43), in which the $\mathfrak{F C}^{k}$. are constant vectors chosen at pleasure. We shall proceed from this standpoint.

It was observed in $\$ 3$ that the conclusions drawn for the vector system (10) are expressible also for the system (5) by means of the relations (12). Hence if the vector relation (38) is resolved into its components, and $y_{1}^{s}(x)$ and $y_{2}^{s}(x)$ are expressed in terms of $y^{s}(x)$ and $y^{s^{\prime}}(x)$, we obtain from it the simultaneous formal expansions

$$
\begin{aligned}
& f_{1}(x) \approx \sum_{s=1}^{\infty} c_{s} y^{s}(x) \\
& f_{2}(x) \approx \sum_{s=1}^{\infty} c_{s}\left\{y^{s^{\prime}}(x)-\lambda r_{1}(x) y^{s}(x)\right\} e^{-\int_{0}^{x} q_{1}(x) d x},
\end{aligned}
$$

associated with the system (5). In these relations $f_{1}(x)$ and $f_{2}(x)$ are arbitrary, while the coefficients $c_{s}$ are the same in both series and are determined by (42).

9. The formal derivation of the contour integral. The characteristic values of the adjoint systems (28) and (29) are the same, and if $\lambda$ is not such a value there exists for the systems a Green's matrix $G(x, t, \lambda)$ in terms of which the solution of the non-homogeneous system corresponding to (29), i.e.,

$$
\begin{aligned}
& \cdot \mathscr{H}(U, \lambda)=\cdot \mathcal{A}(x), \\
& \cdot V_{m n}-V_{h l} W_{h l}^{-1}(\lambda) W_{m n}(\lambda)=\mathcal{O},
\end{aligned}
$$


is given by the formula

$$
V(t)=-\int_{0}^{1} \cdot A(x) G(x, t, \lambda) d x .^{*}
$$

At the characteristic values the elements of $G(x, t, \lambda)$ become infinite, and if, in particular, $G(x, t, \lambda)$ has a simple pole at a given characteristic value the form of its residue there is known and is given by the formula

$$
G^{s}(x, t)=\lim _{\lambda \rightarrow \lambda_{s}} G(x, t, \lambda)\left\{\lambda-\lambda_{s}\right\}=a_{s} \Upsilon^{s}(x) \cdots Z^{s}(t),
$$

where $a_{s}$ is a specific constant. $\dagger$ With these facts at hand we shall seek formally to construct a function with residues which are the terms of the the series (38), and hence to express the sum of any number of terms of this series as a contour integral in the $\lambda$ plane.

Let $\cdot C(x, \lambda)$ be a vector to be subsequently determined, and let $\cdot V(x, \lambda)$ be accordingly defined by the equation

$$
\cdot(x, \lambda)=\cdot Z^{s}(x)+\cdot \mathcal{C}(x, \lambda)\left\{\lambda-\lambda_{s}\right\} .
$$

The substitution of $\cdot Z^{*}(x)$ from this relation into the system (29) with $\lambda=\lambda_{s}$ yields for $\cdot V(x, \lambda)$ the differential system

(a) $\cdot \mathscr{H}(U, \lambda)=\left\{\lambda-\lambda_{s}\right\}\left\{\cdot \mathcal{V}(x, \lambda) R(x)+\cdot \mathscr{H}\left(\cdot \mathcal{C}, \lambda_{s}\right)\right\}$,

(b) $\cdot \mathcal{V}_{m n}-\cdot \mathcal{V}_{h l} \mathscr{W}_{h l}^{-1}(\lambda) \mathscr{W}_{m n}(\lambda)=\left\{\lambda-\lambda_{s}\right\} \cdot \mathcal{H C}\left(\cdot \mathcal{C}, \lambda_{s}, \lambda\right)$,

where

(50) $\cdot \operatorname{FC}\left(\cdot \bigodot, \lambda_{s}, \lambda\right)=\cdot \bigodot_{m n}(\lambda)-\cdot \bigodot_{h l}(\lambda) \mathscr{W}_{h l}^{-1}(\lambda) \mathscr{W}_{m n}(\lambda)-\cdot \mathcal{Z}_{h l} \sum_{k=0}^{K} \frac{\lambda_{s}{ }^{k}}{W_{h l}\left(\lambda_{s}\right)} \mathscr{Q}^{k}(\lambda)$.

The relation (49b) is readily verified with the use of (40), and evidently reduces to the form $(46 \mathrm{~b})$ if

$$
\cdot \mathcal{H C}\left(\cdot \mathcal{C}, \lambda_{s}, \lambda\right) \equiv \mathcal{O} \text {. }
$$

This identity (51), looked upon as a condition on the hitherto undetermined vector $\cdot \mathcal{C}(x, \lambda)$ contains but two component relations, and hence imposes but two conditions on the four elements of $\cdot \mathcal{C}(1, \lambda)$ and $\cdot \mathcal{C}(0, \lambda)$. It is clear, therefore, that $\cdot \mathcal{C}(x, \lambda)$ may always be chosen to satisfy (51). If we assume such a choice the system (49) may be formally considered as of the type (46), and hence we may write

$$
U(t, \lambda)=-\left\{\lambda-\lambda_{s}\right\} \int_{0}^{1}\left\{\cdot U(x, \lambda) R(x)+\cdot \mathscr{H}\left(\cdot \mathcal{C}, \lambda_{s}\right)\right\} G(x, t, \lambda) d x .
$$

* Birkhoff and Langer, loc. cit., pp. 67, 68.

† Birkhoff and Langer, loc. cit., p. 106. 
As $\lambda \rightarrow \lambda$, this relation becomes in virtue of (47) and (48)

$$
\cdot Z^{*}(t)=-a_{s} \int_{0}^{1}\left\{\cdot Z^{s}(x) R(x) \Upsilon^{s}(x) \cdot+\cdot \mathcal{H}\left(\cdot \mathcal{C}, \lambda_{s}\right) \Upsilon^{s}(x) \cdot\right\} d x \cdot Z^{*}(t) .
$$

The elimination of the vector $\mathcal{C}$ from the equality (52) may now be made as follows. From equations (28) and (27) we find that

$$
\begin{aligned}
\int_{0}^{1} \cdot \mathscr{H}\left(\cdot \mathcal{C}, \lambda_{s}\right) \Upsilon^{s}(x) \cdot d x & =\left.\cdot \mathcal{C}\left(x, \lambda_{s}\right) \Upsilon^{s}(x) \cdot\right|_{0} ^{1}=\cdot \mathcal{C}_{h l}\left(\lambda_{s}\right) \Upsilon_{h l}^{s} \cdot+\cdot \mathcal{C}_{m n}\left(\lambda_{s}\right) \Upsilon_{m n}^{s} \\
& =\left\{-\cdot \mathcal{C}_{h l}\left(\lambda_{s}\right) \mathscr{W}_{h l}^{-1}\left(\lambda_{s}\right) \mathscr{W}_{m n}\left(\lambda_{s}\right)+\cdot \mathcal{C}_{m n}\left(\lambda_{s}\right)\right\} \Upsilon_{m n}^{s},
\end{aligned}
$$

while in this final form the quantity within the brace is by (51) equal to the last term on the right of $(50)$ with $\lambda$ replaced by $\lambda_{s}$. Hence we may write (52) in the form

$$
\cdot Z^{s}(t)=-a_{s}\left\{\int_{0}^{1} \cdot Z^{s} R Y^{s} \cdot d x+\cdot Z_{h l}^{s} \sum_{k=0}^{K} \frac{\lambda_{s}{ }^{k}}{W_{h l}\left(\lambda_{s}\right)} Q^{k}\left(\lambda_{s}\right) \Upsilon_{m n}^{s} \cdot\right\} \cdot Z^{s}(t) .
$$

The coefficient of $\cdot Z^{s}(t)$ on the right of this relation is a matrix all of whose elements are the same, and because of this, as is easily verified, we may multiply the relation by $c_{s} \Upsilon^{s}(x)$. on the left and subsequently omit the the vector $\cdot Z^{*}(t)$ on the right. Then replacing the quantity within the brace by its equivalent as given by (42), we find that

$$
c_{s} \Upsilon^{s}(x) \cdot=-a_{s} \Upsilon^{s}(x) \cdot\left\{\int_{0}^{1} \cdot Z^{s}(t) R(t) \mathfrak{F}(t) \cdot d t+\cdot Z_{h l}^{s} K\left(\lambda_{s}\right) \cdot\right\} .
$$

The vector $\cdot Z_{h l}^{s}$ by the definitions (25) is a linear combination of the vectors $\cdot Z^{s}(1)$ and $\cdot Z^{s}(0)$ with coefficients which are constant matrices. In virtue of formula (47), therefore, we may write (53) in the form

$$
\text { (54) } c_{s} \Upsilon^{s}(x) \cdot=-\int_{0}^{1} G^{s}(x, t) R(t) \mathcal{F}(t) \cdot d t-\left\{G^{s}(x, 1) \mathcal{C}_{1}+G^{s}(x, 0) \mathcal{C}_{0}\right\} K\left(\lambda_{s}\right) \cdot
$$

where $C_{0}$ and $C_{1}$ are constant. Now the elements of $K(\lambda)$. were observed in $\$ 8$ to be rational functions of $\lambda$ of degree at most $(-1)$. We shall consider these rational functions expanded in powers of $1 / \lambda$, and for $|\lambda|$ large we shall replace the elements of $K(\lambda)$. by the leading terms of the resulting series. Then if $C_{n}$ is a contour enclosing the first $n$ characteristic values and no others we are led by (54) to the correspondence

$$
\begin{aligned}
\sum_{s=1}^{n} c_{s} \Upsilon^{*}(x) \cdot \sim \frac{-1}{2 \pi i} & \int_{0}^{1} \int_{c_{n}} G(x, t, \lambda) R(t) F(t) \cdot d \lambda d t \\
& -\frac{1}{2 \pi i} \int_{C_{n}}\left\{G(x, 1, \lambda) A_{1} \cdot+G(x, 0, \lambda) \mathcal{A}_{0} \cdot\right\} \frac{d \lambda}{\lambda},
\end{aligned}
$$


where the vectors $\mathcal{A}_{1}$. and $\mathcal{A}_{0}$. are constants, and where in using the symbol $\sim$ in place of the sign of equality we waive the questions of the terms in $K(\lambda)$. which have been dropped and also of the residue introduced by the integrand in this form at $\lambda=0$.

Let the vector $\mathcal{A}(x)$. be defined now by the formula

$$
\mathcal{A}(x) \cdot=x \mathcal{A}_{1} \cdot+(x-1) \mathcal{A}_{0} .
$$

Then recalling the discontinuity of the Green's matrix at the point $t=x$, $^{*}$ we find that

$$
\begin{aligned}
G(x, 1, \lambda) \mathcal{A}_{1} \cdot+G(x, 0, \lambda) \mathcal{A}_{0} \\
=-\mathcal{A}(x) \cdot+\int_{0}^{1}\left\{G_{t}(x, t, \lambda) \mathcal{A}(t) \cdot+G(x, t, \lambda) \mathcal{A}^{\prime}(t) \cdot\right\} d t \\
\quad=-\mathcal{A}(x) \cdot-\int_{0}^{1} G(x, t, \lambda)\left\{\lambda \mathcal{R}(t) \mathcal{A}(t)+\mathrm{B}(t) \mathcal{A}(t) \cdot-\mathcal{A}^{\prime}(t) \cdot\right\} d t
\end{aligned}
$$

It follows that the relation (55) may be written in the form

$$
\begin{aligned}
& \sum_{s=1}^{n} c_{s} Y^{*}(x) \cdot \sim \mathcal{A}(x) . \\
& \quad-\frac{1}{2 \pi i} \int_{0}^{1} \int_{C_{n}} G(x, t, \lambda)\left\{R(t) \bar{F}(t) \cdot-\lambda^{-1} \mathcal{B}(t) \mathcal{A}(t) \cdot+\lambda^{-1} \mathcal{A}^{\prime}(t) \cdot\right\} d \lambda d t
\end{aligned}
$$

where we have abbreviated by setting

$$
\mathscr{F}(t) \cdot-\mathcal{A}(t) \cdot=\overline{\mathfrak{F}}(t) \cdot .
$$

The vector $K(\lambda)$. is given by (44) in terms of the vectors $\mathfrak{F}^{k}$. which we assumed in $\$ 8$ could be chosen at pleasure. Since the coefficients of $\mathcal{A}(x)$. in turn depend upon $K \cdot$, it is clear that we may at this point consider these coefficients at our disposal. We shall choose them so that the vector (57) satisfies the conditions

$$
\overline{\mathfrak{F}}(0) \cdot=\mathcal{O}, \quad \bar{F}(1) \cdot=\mathcal{O},
$$

namely,

$$
\mathcal{A}(x) \cdot=x \mathcal{F}(1) \cdot+(1-x) \mathcal{F}(0) \cdot .
$$

Lastly we shall retain in the brace of the integrand in (56) only the term of highest degree in $\lambda$. This is consistent with our previous disposal of all but the term of highest degree in the vector $K$. The integral to which we are thus led is, then,

\footnotetext{
* Birkhoff and Langer, loc. cit., p. 70.
} 


$$
\mathfrak{J}_{n}(\dot{x}, \mathcal{F} \cdot)=\mathcal{A}(x) \cdot-\frac{1}{2 \pi i} \int_{0}^{1} \int_{C_{n}} G(x, t, \lambda) R(t) \bar{F}(t) \cdot d \lambda d t .
$$

We shall consider in subsequent sections the convergence of this expression (60) as $n \rightarrow \infty$, the contours $C_{n}$ being those described in $\$ 7$. In that discussion we shall ignore the formal deductions above by which the expression $\mathfrak{J}_{n}\left(x, \mathfrak{J}^{\cdot}\right)$ was deduced. This evidently means replacing the formal series (38) by the series of residues of the integrand in (60), and we shall henceforth refer to this series of residues as the expansion associated with the arbitrary vector $F(x) \cdot$. It will ultimately be shown that under suitable conditions this expansion is convergent.

10. The contour integrals for the summability of the expansion. The vector integral (60) may or may not converge as $n \rightarrow \infty$. Even in the case of non-convergence, however, its component integrals, or the corresponding expansions, may be summable by suitable means, and indeed we shall show later that such summability and its order depend directly upon the boundary conditions of the original differential system. The method of summation of series to which we shall resort is described as follows.

The components of the formal vector expansion (38) are given by the formulas

$$
f_{i}(x) \approx \sum_{r=1}^{\infty} c_{r} y_{i}{ }^{r}(x) \quad(i=1,2) .
$$

In connection with them we shall consider the sequence of sums

$$
\sum_{r=1}^{n} c_{r}\left\{1-\left(\frac{\lambda_{r}}{\Lambda_{n}}\right)^{\mu}\right\}^{\sigma_{i}} y_{i}{ }^{r}(x) \quad\left(i=1,2 ; n=n_{1}, n_{2}, \cdots\right),
$$

in which $\Lambda_{n}$ is the radius of the contour $C_{n}$ of $\$ 7$ and $\mu$ is a positive constant whose precise specification we defer to a later section. The index $\sigma_{i}$ is a constant, positive or zero, and for non-integral values of it we define the quantity with exponent $\sigma_{i}$ by the formula

$$
A^{\sigma_{i}}=e^{\sigma_{i} \log A},
$$

where the principal value of the logarithm is to be understood.

The expressions (62) are a form of the Riesz typical means* for the series (61), and if for either value of $i$ the sequence (62) converges the corresponding

\footnotetext{
* The application of the Riesz typical means in this manner to Birkhoff's and Fourier's series has been made by Stone, these Transactions, vol. 28 (1926), pp. 695-761; and vol. 29 (1927), pp. 25-53; also Bulletin of the American Mathematical Society, vol. 33 (1927), pp.721-732. More detailed discussions and references are to be found in these papers.
} 
expansion (61) is said to be summable of order $\sigma_{i}$. The extension of this definition to the vector form is obvious. Thus we shall define the matrix $S_{n}(\lambda)$ by the formulas

$$
\begin{aligned}
& \delta_{n}(\lambda)=\left(\delta_{i} s_{i n}(\lambda)\right), \\
& s_{i n}(\lambda)=\left\{1-\left(\frac{\lambda}{\Lambda_{n}}\right)^{\mu}\right\}^{\sigma_{i}},
\end{aligned}
$$

and shall refer to the expansion (38) as summable of order $\left(\sigma_{1}, \sigma_{2}\right)$, if the sequence of expressions

$$
\sum_{r=1}^{n} c_{r} S_{n}\left(\lambda_{r}\right) \Upsilon^{r}(x)
$$

converges as $n \rightarrow \infty$.

The deduction of a formulation for summability of the vector integral $\mathfrak{J}_{n}(x, \mathfrak{F} \cdot)$ of $(60)$ is now easily made. Thus the general term of the expression (64) is obtainable by multiplying the relation (54) on the left by the matrix $S_{n}\left(\lambda_{r}\right)$. Then since $S_{n}(\lambda)$ is analytic over the entire plane the steps to the relations corresponding to (55) and (56) involve no difficulties, and yield the contour integral related to the sum (64). Precisely the considerations made at the close of $\$ 9$ lead then to a study of the integral

$$
\mathcal{F}_{n}(x, \mathscr{F} \cdot)=\mathcal{A}(x) \cdot-\frac{1}{2 \pi i} \int_{0}^{1} \int_{C_{n}} S_{n}(\lambda) G(x, t, \lambda) R(t) \bar{F}(t) \cdot d \lambda d t .
$$

If this integral converges with particular. values of the constants $\sigma_{i}$ we shall say that the $i$ th component of the integral (60) is summable of order $\sigma_{i}$, or, more briefly, that the expansion $\Im_{n}(x, \mathcal{F} \cdot)$ is summable of order $\left(\sigma_{1}, \sigma_{2}\right)$. Since $S_{n}(\lambda)$ reduces to the unit matrix for $\sigma_{1}=\sigma_{2}=0$, the integral $(60)$ is clearly included in the integral $(65)$, and summability of order $(0,0)$ signifies convergence.

11. The simultaneous expansions associated with system (5). The components of the formal vector expansion (38) were seen in $\$ 8$ to yield the simultaneous formal expansions (45) associated with the differential system (5). The corresponding resolution of the vector relation (60) will in similar manner lead to a pair of contour integrals involving the arbitrary components $f_{1}(x), f_{2}(x)$, of the vector $\Im(x)$. The expression of these integrals in terms solely of quantities associated with the system (5) requires, to begin with, the determination of the relationship between the Green's function $G(x, t, \lambda)$ for system (5), and the elements of the Green's matrix $G(x, t, \lambda)$ for the system (9). 
If in system (7) the second equation is modified by the addition of a function $\phi(x)$ to its right-hand member, the resulting equations are the components of the vector equation

$$
\Upsilon^{\prime}(x) \cdot-\{R(x) \lambda+\mathcal{B}(x)\} \Upsilon(x) \cdot=\left(\begin{array}{c}
0 \\
\phi(x)
\end{array}\right),
$$

with $b_{i}(x)$ and $q_{i}(x)$ related by formulas (11). On the other hand, if $y_{2}(x)$ is eliminated from this modified system (7), it is found that $y_{1}(x)$ satisfies the equation

$$
l\left(y_{1}, \lambda\right)=\phi(x) b_{1}(x),
$$

where $l(y, \lambda)$ is used to denote the left-hand member of equation (5a). Upon adjoining to equation (66) the boundary condition (10b), and to equation (67) the corresponding boundary conditions (5b), we obtain two non-homogeneous differential systems which are solved respectively by the formulas

$$
\begin{gathered}
\Upsilon(x) \cdot=\int_{0}^{1} G(x, t, \lambda)\left(\begin{array}{c}
0 \\
\phi(t)
\end{array}\right) \cdot d t \\
y_{1}(x)=\int_{0}^{1} G(x, t, \lambda) b_{1}(t) \phi(t) d t .
\end{gathered}
$$

Inasmuch as the value of $y_{1}(x)$ given by the first component formula of (68a) must be identical with the value given by the formula (68b), regardless of the choice of the function $\phi(t)$, we conclude that

$$
g_{12}(x, t, \lambda)=G(x, t, \lambda) b_{1}(t) .
$$

To obtain from this last equality the formulas for the remaining elements of the Green's matrix, we recall that in its points of continuity the matrix $G(x, t, \lambda)$ satisfies the equation (9a) as a function of $x$, and satisfies the equation adjoint to $(9 \mathrm{a})$ as a function of $t$; i.e.,

$$
\begin{aligned}
& G_{x}(x, t, \lambda)=\{R(x) \lambda+\mathbb{B}(x)\} G(x, t, \lambda), \\
& G_{t}(x, t, \lambda)=-G(x, t, \lambda)\{R(t) \lambda+\mathbb{B}(t)\} .{ }^{*}
\end{aligned}
$$

From these equations we draw now respectively the component relations

$$
\begin{aligned}
& \frac{\partial g_{12}}{\partial x}=\lambda r_{1}(x) g_{12}+b_{1}(x) g_{22}, \\
& \frac{\partial g_{12}}{\partial t}=-\lambda r_{2}(t) g_{12}-b_{1}(t) g_{11},
\end{aligned}
$$

* Birkhoff and Langer, loc. cit., pp. 69, 70. 
which yield the values of $g_{22}(x, t, \lambda)$ and $g_{11}(x, t, \lambda)$ in terms of the element (69). Lastly, a further component relation of (70a), i.e.,

$$
\frac{\partial g_{11}}{\partial x}=\lambda r_{1}(x) g_{11}+b_{1}(x) g_{21}
$$

yields the value of $g_{21}(x, t, \lambda)$ in terms of the value $g_{11}(x, t, \lambda)$ already at hand. For convenience in summarizing these results, we abbreviate by setting

$$
\bar{G}(x, t, \lambda)=\left\{G_{x}(x, t, \lambda)-\lambda r_{1}(x) G(x, t, \lambda)\right\} e^{-\int_{0}^{x} q_{1} d x} .
$$

Then replacing the function $b_{1}(x)$ by its value as given in (11), we obtain the final formulas

$$
\begin{aligned}
& g_{11}(x, t, \lambda)=-G_{t}(x, t, \lambda)-\left\{\lambda r_{2}(t)+q_{1}(t)\right\} G(x, t, \lambda) \\
& g_{12}(x, t, \lambda)=G(x, t, \lambda) e^{\int_{0}^{t} q_{1} d t} \\
& g_{21}(x, t, \lambda)=-\bar{G}_{t}(x, t, \lambda)-\left\{\lambda r_{2}(t)+q_{1}(t)\right\} \bar{G}(x, t, \lambda), \\
& g_{22}(x, t, \lambda)=\bar{G}(x, t, \lambda) e \int_{0}^{t}{ }^{q_{1} d t} .
\end{aligned}
$$

With the relations thus deduced at hand the components of the integral (60) may now be expressed directly in terms of the differential system (5). Thus we find for them the formulas

$$
\begin{aligned}
& I_{1 n}\left(f_{1}, f_{2}\right)=a_{1}(x)+I_{n}\left(f_{1}, f_{2}, G\right), \\
& I_{2 n}\left(f_{1}, f_{2}\right)=a_{2}(x)+I_{n}\left(f_{1}, f_{2}, \bar{G}\right),
\end{aligned}
$$

where

$$
\begin{array}{r}
I_{n}\left(f_{1}, f_{2}, G\right)=\frac{1}{2 \pi i} \int_{0}^{1} \int_{C_{n}}\left\{G_{t}(x, t, \lambda) r_{1}(t) \bar{f}_{1}(t)+G(x, t, \lambda)\left[\overline { f } _ { 1 } ( t ) \left\{\lambda r_{1}(t) r_{2}(t)\right.\right.\right. \\
\left.\left.\left.+r_{1}(t) q_{1}(t)\right\}-\bar{f}_{2}(t) r_{2}(t) e \int_{0}^{t} q_{1} d t\right]\right\} d \lambda d t .
\end{array}
$$

The convergence or summability of these expansions $I_{1 n}\left(f_{1}, f_{2}\right)$ and $I_{2 n}\left(f_{1}, f_{2}\right)$ to the respective values $f_{1}(x)$ and $f_{2}(x)$, will clearly follow from the argument which we shall develop in later sections to establish the convergence or summability of the vector integral $(60)$ to the value $\mathcal{F}(x) \cdot$.* $^{*}$

* The expansion theorem for a single arbitrary function in terms of the solutions of a system of type (5) is contained in the work of Tamarkin 2 , loc. cit., $\$ 6$, where the expansion formula is derived by methods distinct from those of the present paper. Despite a considerable difference in the final formulations, it is found that the expansion given by him for a function $f_{1}(x)$ is formally included in the first of the expansions (72) above, being deducible from the formula $I_{1 n}\left(f_{1}, f_{2}\right)$ with the particular choice $f_{2}(x)=f_{1}^{\prime}(x) \exp \left(-\int_{0}^{x} q_{1} d x\right)$. The identification of the two results is most easily made by comparing the formulas of Tamarkin 2, pp. 35-36, with the first component relation of (54), the values (71) being substituted and suitable choice of the elements of the matrices $\bigodot_{0}, C_{1}$, and $K$. being made. We note that the argument employed by Tamarkin can also be adapted for application to the differential system in matrix form. When applied in that manner it yields a derivation which may be considered alternative to that above for the expansion of an arbitrary vector. 
12. The Green's matrix. The Green's matrix for the vector system (10) is given by the formula

$$
G(x, t, \lambda)=\frac{1}{2} \Upsilon(x)\left\{ \pm J+\mathscr{D}^{-1}(\lambda)\left\{\mathscr{W}_{12}(\lambda) \Upsilon(0)-\mathscr{W}_{34}(\lambda) \Upsilon(1)\right\}\right\} Z(t),
$$

where the ambiguous sign is to be chosen + when $t<x$, and - when $t>x .^{*}$ In this formula the matrix $J$ is the unit matrix, $\Upsilon(x)$ and $Z(x)$ are any pair of associated matrix solutions of the equation (9a) and its adjoint equation respectively, and $\mathcal{D}(\lambda)$ is the left-hand member of equation (9b). The substitution in formula (73) of the values of $\Upsilon(x)$ and $Z(t)$ given by the relations (16) and (17) leads to the explicit evaluation of $G(x, t, \lambda)$. We find it convenient to write the result of this computation in the form

$$
G(x, t, \lambda)=\sum_{l=0}^{3} G^{l}(x, t, \lambda),
$$

where, in terms of the matrices (23) and the determinants (31) and (34), we have the formulas

(a) $\quad G^{0}(x, t, \lambda)=\mathscr{P}(x, \lambda) J_{11} 2(t, \lambda) D^{-1}(\lambda) \bar{D}(\lambda) e^{\lambda R_{1}(x, t)}$,

$$
\text { where } \bar{D}(\lambda)=D_{0}(\lambda)+D_{2}(\lambda) e^{\lambda R_{2}}- \begin{cases}0 & \text { for } t \leqq x, \\ D(\lambda) & \text { for } t>x ;\end{cases}
$$

(b) $\quad G^{1}(x, t, \lambda)=P(x, \lambda) \mathfrak{J}_{22} 2(t, \lambda) D^{-1}(\lambda) \bar{D}(\lambda) e^{\lambda R_{2}(x, t)}$,

$$
\text { where } \bar{D}(\lambda)=D_{0}(\lambda)+D_{1}(\lambda) e^{\lambda R_{1}}- \begin{cases}0 & \text { for } t \leqq x \\ D(\lambda) & \text { for } t>x ;\end{cases}
$$

(c) $\quad G^{2}(x, t, \lambda)=\mathscr{P}(x, \lambda) J_{12} 2(t, \lambda) D^{-1}(\lambda) D_{4}(\lambda) e^{\lambda R_{1}(x)+\lambda R_{2}(1, t)}$;

(d) $\quad G^{3}(x, t, \lambda)=P(x, \lambda) J_{21} 2(t, \lambda) D^{-1}(\lambda) D_{5}(\lambda) e^{\lambda R_{2}(x)+\lambda R_{1}(1, t)}$.

For convenience in substituting these terms in the integral (65), we note further that

$$
S_{n}(\lambda) P(x, \lambda) J_{h l} 2(t, \lambda) R(t) \overline{\mathcal{F}}(t) \cdot=\left(s_{i n}(\lambda) \lambda^{\delta_{i h^{-1}}} \omega_{i, h l}(x, t, \lambda)\right) \cdot
$$

where it is found from the formulas (16c) and (17b) that

$$
\omega_{i, h l}(x, t, \lambda)=p_{i h}(x) r_{l}(t) \bar{f}_{l}(t)+\lambda^{-1}\left\{\left[\alpha_{i, h l}^{(1)}(x, t)\right] \bar{f}_{1}(t)+\left[\alpha_{i, h l}^{(2)}(x, t)\right] \bar{f}_{2}(t)\right\},
$$

the functions $\alpha_{i, h l}^{(j)}(x, t)$ being expressible in terms of the quantitites [ $p_{i j}(x)$ ] and $\left[q_{i j}(t)\right]$.

13. The specification of the value $\mu$. The introduction of the factors $\left\{1-\alpha_{n} / \omega\right\} \cdot$ into the partial sums

* Birkhoff and Langer, loc. cit., p. 110. 


$$
S_{N}(x)=\sum_{n=1}^{N} c_{n} y_{n}(x)
$$

of an infinite series, to form the Riesz typical means

$$
\sum_{n=1}^{N}\left\{1-\frac{\alpha_{n}}{\omega}\right\}^{\sigma} c_{n} y_{n}(x), \quad \alpha_{N}<\omega \leqq \alpha_{N+1},
$$

is motivated by the purpose, usual in schemes of summation, of assigning varying weights to the sums $S_{N}{ }^{*}$ From this point of view the quantities $\alpha_{n} / \omega$ are naturally specified by the rôle assigned them to be real and positive. Without adhering strictly to this viewpoint we shall nevertheless utilize it as a guide in the choice of the hitherto unspecified constant $\mu$ of formulas (62) and (63).

Consider first the case in which the constant $\alpha$ of $(6 \mathrm{~b})$ is $\pi / 2$. By $\$ 6$ the characteristic values are confined then to strips of asymptotically constant width, bounded by curves which approach parallelism with the axes of reals. Hence for any sufficiently large value of $j$ the quantity $\arg \lambda_{j}$ lies arbitrarily near one of the values 0 and $\pi$, and since $\Lambda_{n}$ is real, it follows that as $n$ and $N$ become infinite, $n \leqq N$, the quantity $\left\{1-\left(\lambda_{n} / \Lambda_{N}\right)^{\mu}\right\}$ is asymptotically real and positive if $\mu$ is a multiple of 2 . We shall, therefore, choose $\mu=2$ when $\alpha=\pi / 2$.

If $\alpha=0$ the characteristic values, by $\S 6$, lie in strips which approach parallelism with the axes of imaginaries. Hence in this case the value $\arg \boldsymbol{\lambda}_{j}$ for any $j$ sufficiently large lies near one of the values $\pi / 2$ and $3 \pi / 2$, and it follows that $\left\{1-\left(\lambda_{n} / \Lambda_{N}\right)^{\mu}\right\}$ is now asymptotically real and positive if $\mu$ is a multiple of 4 . We are led, therefore, to the choice $\mu=4$ when $\alpha=0$.

In the case $0<\alpha<\pi / 2$ the strips containing the characteristic values approach parallelism with certain other rays in the $\lambda$ plane, these rays being four in number, and being given by the formulas (37) if no term of the characteristic equation (35) vanishes identically. We shall confine our considerations, when $0<\alpha<\pi / 2$, to the case in which this hypothesis on the equation (35) is fulfilled.

If, in formula (63), $\sigma_{i}=0$ for either value of $i$, the element $s_{i n}(\lambda)$ reduces to unity and the question of the value of $\mu$ is obviated so far as the corresponding component of (65) is concerned. This corresponds, of course, to the convergence of the respective component of the expansion (60). If $\sigma_{i}>0$, on

\footnotetext{
1915, p. 22.

* Cf. Hardy and Riesz, The General Theory of Dirichlet's Series, Cambridge University Press,
} 
the other hand, $\mu$ must be chosen, and the principle followed in the previous cases clearly demands now a choice such that

$$
\mu(\pi / 2 \pm \alpha) \equiv 0
$$

$(\bmod 2 \pi)$,

for both determinations of the ambiguous sign involved. It is found directly that the relations (78) are possible only if $\alpha$ is commensurable with $\pi$, and hence we shall restrict our further considerations when $\sigma_{i}>0$ to this case.* The values of $\mu$ which satisfy (78) are then readily determined to be even integers, and we shall choose $\mu$ as the smallest positive value for which the relation (78) holds.

14. Auxiliary theorems. The proof of the convergence of the integral (65) as $n \rightarrow \infty$ is to be based upon certain classical theorems of Lebesgue and upon certain partial results which we shall summarize in the form of lemmas. For convenience in reference we group these theorems and lemmas together at this point.

Consider the integral

$$
I(\psi, n)=\int_{a}^{b} \psi(z) \phi(z, n) d z,
$$

where $\phi(z, n)$ is integrable with respect to $z$ for the values $n$ of a sequence $n_{1}, n_{2}, \cdots$; where $n_{s} \rightarrow \infty$, as $s \rightarrow \infty$. We quote the following two theorems. $\dagger$

Lebesgue Theorem A. A necessary and suficient condition that

$$
\lim _{n \rightarrow \infty} I(\psi, n)=0,
$$

for every integrable function $\psi(z)$, is that

$$
\begin{aligned}
& |\phi(z, n)|<M, a \text { constant independent of } z \text { and } n ; \\
& \lim _{n \rightarrow \infty} \int_{z_{1}}^{z_{2}} \phi(z, n) d z=0, \quad \text { for } a \leqq z_{1} \leqq z_{2} \leqq b .
\end{aligned}
$$

Lebesgue Theorem B. A necessary and sufficient condition that

$$
\lim _{n \rightarrow \infty} I(\psi, n)=c \psi(a+),
$$

for every function $\psi(z)$ which is of bounded variation on $(a, b)$, is that

$$
\left|\int_{z_{1}}^{z_{2}} \phi(z, n) d z\right|<M, \text { for } a \leqq z_{1} \leqq z_{2} \leqq b,
$$

* This hypothesis is fulfilled in the cases to which the "means" have been applied by Stone, loc. cit. It is, of course, also satisfied when $\alpha=0$ or $\alpha=\pi / 2$.

† Lebesgue, Annales de Toulouse, (3), vol. 1 (1909), p. 52 and p. 70. 


$$
\begin{array}{ll}
\lim _{n \rightarrow \infty} \int_{z_{1}}^{z_{2}} \phi(z, n) d z=0, & \text { for } \quad a<z_{1} \leqq z_{2} \leqq b, \\
\lim _{n \rightarrow \infty} \int_{a}^{z_{1}} \phi(z, n) d z=c, & \text { for } a<z_{1} \leqq b .
\end{array}
$$

In the following lemmas the variable $z$, which is considered real, is to range as specified either over the interval $\left(0, z_{2}\right)$ or over the interval $\left(z_{1}, z_{2}\right)$ where $z_{1}>0$. The complex parameter $\rho$ is considered to range over the circles of the sequence $C_{n}$ with centers at $\rho=0$ and radii $\Lambda_{n}$, the argument $\chi$ being defined by the relation

$$
\rho=i \Lambda_{n} e^{i x} .
$$

Any arc of $C_{n}$ which lies entirely on the half-plane $0 \leqq \chi \leqq \pi$, i.e., on the half-plane in which $\operatorname{Re}(\rho) \leqq 0$, is designated by $c_{n}$. The symbol $E(z, \rho)$ is used to represent a function which is integrable and bounded for $|\rho|$ sufficiently large, and $M$ is used to indicate a positive constant suitably chosen. Lastly, we use the symbols $O(1)$ and $o(1)$ to indicate functions which are bounded or approach zero respectively as $n \rightarrow \infty$.

LEMMA I.* If $\psi(z)$ is any function which is integrable on the interval $\left(0, z_{2}\right)$ and $0 \leqq z^{\prime} \leqq z^{\prime \prime} \leqq z_{2}$, then

$$
\int_{z^{\prime}}^{z^{\prime \prime}} \int_{c_{n}} e^{\rho z} E(z, \rho) \psi(z) \frac{d \rho}{\rho} d z=o(1) .
$$

LEMмA II. If on the $\operatorname{arc} c_{n}, \chi \leqq \chi_{2}$, then

$$
\int_{c_{n}}\left\{1-e^{i \mu x}\right\}^{\sigma} \rho^{\tau} E(z, \rho) d \rho=\Lambda_{n}{ }^{r+1} \chi_{2}{ }^{\sigma+1} O(1) .
$$

This conclusion is immediately drawn upon using the fact that

$$
\left|1-e^{i \mu x}\right|^{\sigma} \leqq M \chi^{\sigma} \text {. }
$$

LEMMA III. $\dagger$ If $\mu$ is an even integer and $z>0$, the integral

$$
I(z, n)=\int_{c_{n}}\left\{1-e^{i \mu x}\right\}^{\sigma} \rho^{\tau} e^{\rho z} d \rho
$$

satisfies the relation

$$
I(z, n)=z^{-\sigma-1} \Lambda_{n}{ }^{r-\sigma} O(1) .
$$

On the $\operatorname{arc} c_{n}$ we have clearly

* Tamarkin 2, loc. cit., p. 43.

$\dagger$ Stone, these Transactions, vol. 28 (1926), p. 714. 


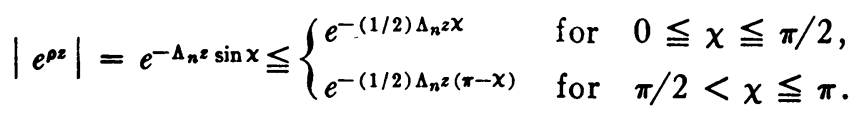

Consider first the integral $I^{\prime}(z, n)$ obtained by replacing $c_{n}$ in (80) by that portion of $c_{n}$ on which $\chi \leqq \pi / 2$. We obtain then from (79) and (81) the inequality

and setting

$$
\left|I^{\prime}(z, n)\right| \leqq M \int_{0}^{\pi / 2} \chi^{\sigma} \Lambda_{n}{ }^{r+1} e^{-(1 / 2) \Delta_{n} 2 x} d \chi
$$

we find that

$$
\zeta=\frac{1}{2} \Lambda_{n} z \chi
$$

$$
\left|I^{\prime}(z, n)\right| \leqq M\left(\frac{2}{z \Lambda_{n}}\right)^{\sigma+1} \Lambda_{n}{ }^{\tau+1} \int_{0}^{\infty} \zeta^{\sigma} e^{-\zeta} d \zeta=M_{1} z^{-\sigma-1} \Lambda_{n}{ }^{r-\sigma} .
$$

The integral $I^{\prime \prime}(z, n)$ contributed to (80) by the integration over the portion of $c_{n}$ for which $\chi>\pi / 2$ may be treated similarly. Thus if we set $\pi-\chi=\eta$, then since $\mu$ is by hypothesis an even integer we have

$$
e^{i \mu x}=e^{-i \mu \eta},
$$

and the reasoning above may be repeated with $-\eta$ replacing $\chi$. This establishes the lemma.

LEMMA IV. If in the expression (80) $\sigma \geqq \tau$, then for any integrable function $\psi(z)$ and any expression $[\alpha(z)]$ of the type (15) with integrable coefficients

$$
\int_{z^{\prime}}^{z^{\prime \prime}} I(z, n) \psi(z)[\alpha(z)] d z=o(1), \text { for } z_{1} \leqq z^{\prime} \leqq z^{\prime \prime} \leqq z_{2}
$$

From the definition (15) we derive the form

$$
\psi(z) \rho^{\tau}[\alpha(z)]=P_{\tau}(z, \rho)+\frac{E(z, \rho)}{\rho} \psi(z),
$$

where $P_{\tau}$ is a polynomial in $\rho$ of degree $\tau$. By Lemma $I$ the last term of (83) contributes to the integral (82) a quantity of order $o(1)$. To discuss the terms of (82) due to the expression $P_{\tau}(z, \rho)$ we observe that Lemma III may be applied to the members on the right of the equality

$$
\int_{z^{\prime}}^{z^{\prime \prime}} I(z, n) d z=\int_{c_{n}}\left\{1-e^{i \mu x}\right\}^{\sigma} \rho^{\tau-1} e^{\rho z^{\prime \prime}} d \rho-\int_{c_{n}}\left\{1-e^{i \mu x}\right\}^{\sigma} \rho^{\tau-1} e^{\rho z^{\prime}} d \rho,
$$

to show that this integral is of order $o(1)$, and hence that we may apply the Lebesgue theorem $A$ to the integrals arising from the terms of the polynomial $P_{r}(z, \rho)$. The conclusion of the lemma follows. 
LEMMA V. If in the integral (80) $\tau=0$, and $c_{n}$ is the semicircle $0 \leqq \chi \leqq \pi$, then

$$
\lim _{n \rightarrow \infty} \int_{0}^{z_{3}} I(z, n) \psi(z) d z=-\pi i \psi(0+)
$$

for any function $\psi(z)$ which is integrable and is of bounded variation in some interval $(0, \epsilon)$ where $\epsilon>0$.

The integration from $\epsilon$ to $z_{2}$ yields a result which is of the order $o(1)$ by Lemma IV. Hence to prove the present lemma we need only show that the integral from 0 to $\epsilon$ satisfies the hypotheses of the Lebesgue theorem $\mathbf{B}$. The first of these hypotheses, (i), is clearly fulfilled, and, applying Lemma III to the integrals on the right of (84) with $\tau=0$, it is also clear that condition (ii) is fulfilled. Lastly, if we set $\phi=e^{i x}$, and recall that $\mu$ is an even integer, we obtain the formula*

$$
I(z, n)=-\int_{-1}^{1}\left\{1-\phi^{\mu}\right\}^{\sigma} e^{i \Lambda_{n} z \phi} i \Lambda_{n} d \phi=-2 i \int_{0}^{1}\left\{1-\phi^{\mu}\right\}^{\sigma} \Lambda_{n} \cos \Lambda_{n} z \phi d \phi,
$$

from which it follows that

$$
\int_{0}^{\epsilon} I(z, n) d z=-2 i \int_{0}^{1}\left\{1-\phi^{\mu}\right\}^{\sigma} \frac{\sin \Lambda_{n} \epsilon \phi}{\phi} d \phi .
$$

But this last integral approaches the limit $-\pi i$, as one sees by applying it to the Lebesgue theorem B itself, and using the Dirichlet integral formula

$$
\lim _{n \rightarrow \infty} \int_{0}^{1} \frac{\sin \Lambda_{n} \epsilon \phi}{\phi} d \phi=\pi / 2 \text {. }
$$

Hence condition (iii) is also fulfilled and the lemma is proved.

15. The integrals $\mathcal{F}_{n}{ }^{0}(x)$. and $\mathcal{F}_{n}{ }^{1}(x) \cdot$ for $0<x<1, \alpha=0$, and $\sigma_{i} \geqq 0$. Let the integrals $\mathcal{F}_{n}^{l}(x) \cdot$ be defined by the formula

$$
\mathcal{F}_{n}^{l}(x) \cdot=\frac{-1}{2 \pi i} \int_{0}^{1} \int_{C_{n}} S_{n}(\lambda) G^{l}(x, t, \lambda) R(t) \bar{F}(t) \cdot d \lambda d t \quad(l=0,1,2,3) .
$$

Then by (74) the sum of these integrals yields the integral in (65) and we may determine the limit of the latter by considering separately the evaluations of the expressions (85). The details of these evaluations differ somewhat in the cases $\alpha=0, \alpha=\pi / 2$, and $0<\alpha<\pi / 2$. They depend also upon whether $x$ lies in the interior or at the ends of the interval $(0,1)$. We begin by considering in detail the case in which $\alpha=0$ and $0<x<1$.

† Cf. Stone loc. cit., pp. 724-726. 
If $\alpha=0$, the constants $\nu_{1}$ and $\nu_{2}$ in (6b) are both unity and the functions $r_{1}(x), r_{2}(x)$, are accordingly real and positive. By $\S 13$ we choose in this case $\mu=4$. Moreover, the deductions which follow require that we add to our hypotheses the condition that in the characteristic equation (35)

$$
D_{0}(\lambda) \not \equiv 0, \quad D_{3}(\lambda) \not \equiv 0
$$

From the formula for $\bar{D}(\lambda)$ given in (75a) we find the alternative forms

$$
D^{-1} \bar{D}\left\{\begin{array}{l}
=\frac{D_{0}+D_{2} e^{\lambda R_{2}}}{D} \\
=1-\frac{D_{1} e^{\lambda R_{1}}+D_{3} e^{\lambda R_{1}+\lambda R_{2}}}{D}, \text { for } t \leqq x,
\end{array}\right.
$$

while for $t>x$ these values are to be modified by subtracting 1 . Now it was observed in $\S 7$ that on the contours $C_{n}$ the quotient of $D(\lambda)$ by any one of its terms is uniformly bounded from zero. Hence we have, in particular, the two evaluations

$$
D^{-1}(\lambda)\left\{\begin{array}{l}
=D_{3}^{-1}(\lambda) e^{-\lambda R_{1}-\lambda R_{2}} O(1) \\
=D_{0}^{-1}(\lambda) O(1)
\end{array}\right.
$$

Let $h$ be defined now as the largest of the numbers $\kappa_{2}-\kappa_{3}, \kappa_{1}-\kappa_{0}$, and $\pm\left(\kappa_{3}-\kappa_{0}\right)$, where for any $l$ the number $\kappa_{j}-\kappa_{l}$ is to be considered zero if $D_{i}(\lambda) \equiv 0$. Then substituting the values (88) in the respective formulas (87), supposing $\operatorname{Re}(\lambda) \leqq 0$ in the first formula and $\operatorname{Re}(\lambda)>0$ in the second, we find that

$$
D^{-1} \bar{D} \begin{cases}=\lambda^{h} e^{-\lambda R_{1}} O(1), & \text { for } \operatorname{Re}(\lambda)>0, \\ =1-\lambda^{h} e^{\lambda R_{1}} O(1), & \text { for } \operatorname{Re}(\lambda) \leqq 0, \quad t \leqq x,\end{cases}
$$

the values to be diminished by one when $t>x$.

With the value of $G^{0}(x, t, \lambda)$ obtained from (75a) with the use of (89) and (76) we may now write the integral (85) for $l=0$ in the explicit form

$$
\begin{aligned}
\mathcal{F}_{n}{ }^{0}(x) \cdot=\frac{-1}{2 \pi i} & \left(\int_{0}^{x} \int_{C_{n^{\prime}}} s_{i n}(\lambda) \lambda^{-\delta_{i 2} \omega_{i, 11}}(x, t, \lambda) e^{\lambda R_{1}(x, t)} d \lambda d t\right. \\
& -\int_{x}^{1} \int_{C_{n^{\prime \prime}}} s_{i n}(\lambda) \lambda^{-\delta_{i 2}} \omega_{i, 11}(x, t, \lambda) e^{\lambda R_{1}(x, t)} d \lambda d t \\
& -\int_{0}^{1} \int_{C_{n^{\prime}}{ }_{i n} \lambda^{h-\delta_{i 2}} \omega_{i, 11} e^{\lambda R_{1}(x)+\lambda R_{1}(1, t)}} O(1) d \lambda d t \\
& \left.+\int_{0}^{1} \int_{C_{n^{\prime \prime}}{ }^{\prime \prime}} s_{i n} \lambda^{h-\delta_{i 2} \omega_{i, 11}} e^{-\lambda R_{1}(1, x)-\lambda R_{1}(t)} O(1) d \lambda d t\right) .
\end{aligned}
$$


where $C_{n}^{\prime}$ is the part of the contour $C_{n}$ on which $\operatorname{Re}(\lambda) \leqq 0$ and $C_{n}^{\prime \prime}$ is the part on which $\operatorname{Re}(\lambda)>0$.

Consider first the final integral on the right of this equation (90). The quantity $\left\{R_{1}(1, x)+R_{1}(t)\right\}$ is positive for all values of $t$ on the interval $(0,1)$. Hence it may assume the rôle of the variable $z$ in Lemma IV, and if we substitute for $\omega_{i, 11}(x, t, \lambda)$ its value as given by (77) it becomes obvious that the lemma in question applies, and hence that the integral is of the order $o(1)$ provided $\sigma_{i} \geqq h-\delta_{i 2}$. A similar argument with the same condition on $\sigma_{i}$ shows that the integral preceding that just considered is likewise of the order $o(1)$.

Consider now the leading integral on the right of (90). In the case of the component $i=2$ the integral is of the type discussed by Lemma I, the rôle of the variable $z$ being taken by the quantity $R_{1}(x, t)$, and it follows, therefore, by Lemma $I$ that the integral is of order $o(1)$. This argument, moreover, may be repeated for the component $i=1$ provided the first term of the expression $\omega_{1,11}(x, t, \lambda)$ as given by (77) is tentatively omitted from the consideration. The discussion of the second integral in (90) is entirely analogous to that of the first, and hence recalling the omitted first term of $\omega_{1,11}$ we find that formula $(90)$ reduces to the form

$$
\begin{aligned}
\mathcal{F}_{n}{ }^{0}(x) \cdot=\frac{-1}{2 \pi i} & \left(\delta_{i 1} \int_{0}^{x} \int_{C_{n^{\prime}}} s_{1 n}(\lambda) \bar{f}_{1}(t) e^{\lambda R_{1}(x, t)} r_{1}(t) d \lambda d t\right. \\
& \left.-\delta_{i 1} \int_{x}^{1} \int_{C_{n^{\prime}}{ }^{\prime \prime}} s_{1 n}(\lambda) \bar{f}_{1}(t) e^{\lambda R_{1}(x, t)} r_{1}(t) d \lambda d t+o(1)\right) .
\end{aligned}
$$

To reveal more clearly the structure of the integrals in this formula we resort to the change of variable defined by the relations

$$
R_{1}(t)=\xi_{0}-\xi, \quad R_{1}(x)=\xi_{0}, \quad R_{1}=\xi_{0}-\xi, \quad \bar{f}_{1}(t)=\psi_{1}\left(\xi_{0}-\xi\right) .
$$

Substituting these values and subsequently replacing $\xi$ and $\lambda$ in the second integral by $-\xi$ and $-\lambda$, respectively, we find for (91) the form

$$
\begin{aligned}
\mathcal{F}_{n}{ }^{0}(x) \cdot=\frac{-1}{2 \pi i} & \left(\delta_{i 1} \int_{0}^{\xi_{0}} \int_{C_{n^{\prime}}} s_{1 n}(\lambda) e^{\lambda \xi} \psi_{1}\left(\xi_{0}-\xi\right) d \lambda d \xi\right. \\
& \left.+\delta_{i 1} \int_{0}^{\xi_{1}} \int_{C_{n^{\prime}}} s_{1 n}(\lambda) e^{\lambda \xi} \psi_{1}\left(\xi_{0}+\xi\right) d \lambda d \xi+o(1)\right) .
\end{aligned}
$$

Let us suppose now that $x$ is a point in some neighborhood of which $\bar{f}_{1}(t)$ is of bounded variation. Then it is clear from (92) that $\psi_{1}\left(\xi_{0} \pm \xi\right)$ is of bounded variation in some neighborhood of $\xi=0$, and by Lemma $V$ the 
integrals in (93) converge respectively to the values $-\pi i \psi_{1}\left(\xi_{0} \pm \xi\right)$. Since $\psi_{1}\left(\xi_{0} \pm \xi\right) \equiv \vec{f}_{1}(x \pm 0)$, we have the result

$$
\mathcal{F}_{n}{ }^{0}(x) \cdot \rightarrow \frac{1}{2}\left(\begin{array}{c}
\bar{f}_{1}(x-)+\bar{f}_{1}(x+) \\
0
\end{array}\right) .
$$

The discussion thus given for the integral (85) with $l=0$ may be repeated almost without change for the integral with $l=1$. We find from such a discussion that if $x$ is a point in some neighborhood of which $\bar{f}_{2}(t)$ is of bounded variation, and if $\sigma_{i} \geqq h_{1}-\delta_{i 1}$, where $h_{1}$ designates the largest of the numbers $\kappa_{1}-\kappa_{3}, \kappa_{2}-\kappa_{0}, \pm\left(\kappa_{3}-\kappa_{0}\right)$, then

$$
\mathcal{F}_{n}{ }^{1}(x) \cdot \rightarrow \frac{1}{2}\left(\begin{array}{c}
0 \\
\bar{f}_{2}(x-)+\bar{f}_{2}(x+)
\end{array}\right) .
$$

Combining these results and recalling the relation between the vectors $\bar{F}(x) \cdot$ and $\mathcal{F}(x) \cdot$, as given by $(57)$, we obtain finally the result

$$
\mathcal{A}(x) \cdot+\overline{\mathcal{F}}_{n}{ }^{0}(x) \cdot+\mathcal{F}_{n}{ }^{1}(x) \cdot \rightarrow \frac{1}{2} \mathcal{F}(x+) \cdot+\frac{1}{2} \mathfrak{F}(x-) \cdot
$$

16. The integrals $\mathcal{F}_{n}{ }^{2}(x)$ and $\mathcal{F}_{n}{ }^{3}(x)$. for $0<x<1, \alpha=0$, and $\sigma_{i} \geqq 0$. The integrals (85) for $l=2,3$ are now easily evaluated by the method of the preceding section. Thus we have

$$
D^{-1} D_{4}\left\{\begin{array}{lll}
=\lambda^{k_{4}-\kappa_{4}} e^{-\lambda R_{1}-\lambda R_{2}} O(1), & \text { for } & \operatorname{Re}(\lambda)>0, \\
=\lambda^{\kappa_{4}-\kappa_{0}} O(1), & \text { for } & \operatorname{Re}(\lambda) \leqq 0,
\end{array}\right.
$$

and we find in the manner now familiar that

$$
\begin{aligned}
& \mathcal{F}_{n}{ }^{2}(x) \cdot=\frac{-1}{2 \pi i}\left(\int_{0}^{1} \int_{C_{n}{ }^{\prime}} s_{i n}(\lambda) \lambda^{\alpha_{4}-\alpha_{0}-\delta i 2} \omega_{i, 12}(x, t, \lambda) e^{\lambda R_{1}(x)+\lambda R_{2}(1, t)} O(1) d \lambda d t\right. \\
& +\int_{0}^{1} \int_{C_{n}^{\prime \prime}} s_{i n}(\lambda) \lambda^{4_{4}-k_{4}-\delta_{i 2}} \omega_{i, 12}(x, t, \lambda) e^{-\lambda R_{1}(1, x)-\lambda R_{2}(t)} O(1) d \lambda d t .
\end{aligned}
$$

An application of Lemma IV yields the result that these integrals are both of the order $o(1)$ provided $\sigma_{i} \geqq \kappa_{4}-\kappa_{0}-\delta_{i 2}, \kappa_{4}-\kappa_{3}-\delta_{i 2}$. In entirely similar fashion it is found that

$$
\mathcal{Z}_{n}{ }^{3}(x) \cdot \rightarrow \mathcal{O}
$$

provided $\sigma_{i} \geqq \kappa_{5}-\kappa_{0}-\delta_{i 1}, \kappa_{5}-\kappa_{3}-\delta_{i 1}$, and the results of this and the preceding section may, therefore, be summarized as follows:

Theorem 1. If $\alpha=0$ and $D_{0}(\lambda) D_{3}(\lambda) \neq 0$, let $\bar{\kappa}_{i}, i=1,2$, designate the largest of the numbers

$$
\begin{aligned}
& \pm\left(\kappa_{3}-\kappa_{0}\right), \quad \kappa_{2}-\kappa_{3}-\delta_{i 2}, \quad \kappa_{1}-\kappa_{0}-\delta_{i 2}, \quad \kappa_{4}-\kappa_{3}-\delta_{i 2}, \kappa_{4}-\kappa_{0}-\delta_{i 2}, \\
& \kappa_{1}-\kappa_{3}-\delta_{i 1}, \kappa_{2}-\kappa_{0}-\delta_{i 1}, \kappa_{5}-\kappa_{3}-\delta_{i 1}, \kappa_{5}-\kappa_{0}-\delta_{i 1} \text {, }
\end{aligned}
$$


the number $\kappa_{j}-\kappa_{l}$ being considered zero for any $l$ if $D_{j}(\lambda) \equiv 0$. Then with $\mu=4$ and $\sigma_{i}$ chosen in any way subject to the relation $\sigma_{i} \geqq \bar{\kappa}_{i}, i=1,2$, the expansion (60) associated with an arbitrary integrable vector $F(x) \cdot$ is summable of order $\left(\sigma_{1}, \sigma_{2}\right)$ to the value

$$
\frac{1}{2}\{F(x+) \cdot+F(x-) \cdot\},
$$

at every point $x$ within the interval $(0,1)$, in some neighborhood of which $F(x) \cdot$ is of bounded variation.

Corollary 1. If $\bar{\kappa}_{i}=0, i=1,2$, the expansion (60) is convergent.

COROLlaRY 2. For an arbitrary choice of the integrable functions $f_{1}(x)$, $f_{2}(x)$, the expansion $I_{i, n}\left(f_{1}, f_{2}\right)$ given by $(72)$ for either $i=1$, or $i=2$, is summable of order $\sigma_{i} \geqq \bar{\kappa}_{i}$ to the value

$$
\frac{1}{2}\left\{f_{i}(x+)+f_{i}(x-)\right\}
$$

at any point within the interval $(0,1)$, in some neighborhood of which $f_{i}(x)$ is of bounded variation.

In the following section we shall establish the summability of the expansion (in contradistinction to its actual convergence) without hypothesizing the boundedness of the variation of $F(x) \cdot$. In this respect, therefore, the statement of Theorem 1 may be broadened.

17. The expansion (60) when $0<x<1, \alpha=0$ and $\sigma_{i}>0$. It follows from Lemma IV, since the function $\psi_{1}\left(\xi_{0} \pm \xi\right)$ of $\$ 15$ is integrable, that with an arbitrary choice of a positive $\epsilon$, and $\xi>\epsilon$,

$$
\int_{0}^{\xi} \int_{C_{n^{\prime}}} s_{1 n}(\lambda) e^{\lambda \xi} \psi_{1}\left(\xi_{0} \pm \xi\right) d \lambda d \xi=o(1) .
$$

Hence we may also write the formula (93) in the form

$$
\mathcal{F}_{n}{ }^{0}(x)=\frac{-1}{2 \pi i}\left(\delta_{i 1} \int_{0}^{e} I(\xi, n)\left\{\psi_{1}\left(\xi_{0}-\xi\right)+\psi_{1}\left(\xi_{0}+\xi\right)\right\} d \xi+o(1)\right)
$$

where we have set

$$
I(\xi, n)=\int_{C_{n^{\prime}}} s_{1 n}(\lambda) e^{\lambda \xi} d \xi .
$$

This integral $I(\xi, n)$ is of the form considered in Lemmas II and III with $\tau=0$, and we draw from these lemmas the inequalities

$$
\begin{aligned}
& |I(\xi, n)|<M \Lambda_{n}, \\
& |I(\xi, n)|<M \Lambda_{n}{ }^{-\sigma_{1}} \xi^{-\sigma_{1}-1}, \text { for } \xi>0,
\end{aligned}
$$

$M$ being a suitable positive constant. 
Let $\Psi_{1}\left(\xi_{0}, \xi\right)$ and $\Phi_{1}\left(\xi_{0}, \xi\right)$ be defined now by the relations

$$
\begin{aligned}
& \Psi_{1}\left(\xi_{0}, \xi\right)=\psi_{1}\left(\xi_{0}-\xi\right)+\psi_{1}\left(\xi_{0}+\xi\right)-2 \psi\left(\xi_{0}\right), \\
& \Phi_{1}\left(\xi_{0}, \xi\right)=\frac{1}{\xi} \int_{0}^{\xi}\left|\Psi_{1}\left(\xi_{0}, \xi\right)\right| d \xi .
\end{aligned}
$$

Then by a theorem of Lebesgue* the function $\Phi_{1}\left(\xi_{0}, \xi\right)$ approaches zero with $\xi$ for almost all values of $\xi_{0}$. We except from consideration the values of $\xi_{0}$, if any, for which this is not true. Then with an arbitrary choice of an $\eta>0$ the $\epsilon$ in formula (97) may be chosen so that

$$
\Phi_{1}\left(\xi_{0}, \xi\right)<\eta, \text { for } 0 \leqq \xi \leqq \epsilon .
$$

Let the formula (97) be written now in the form

$$
\mathcal{F}_{n}{ }^{0}(x) \cdot=\frac{-1}{2 \pi i}\left(2 \psi\left(\xi_{0}\right) \delta_{i 1} \int_{0}^{e} I(\xi, n) d \xi+\delta_{i 1} \int_{0}^{e} I(\xi, n) \Psi_{1}\left(\xi_{0}, \xi\right) d \xi+o(1)\right)
$$

From Lemma $\mathrm{V}$ we find directly for the first integral in this formula the value

$$
\int_{0}^{e} I(\xi, n) d \xi=-\pi i+o(1) .
$$

The second integral, on the other hand, we evaluate by the following method which is due to Hardy. $\dagger$

From the first of formulas (98), together with (99), we find that for $n$ sufficiently large

$$
\left|\int_{0}^{1 / \Delta_{n}} I(\xi, n) \Psi_{1}\left(\xi_{0}, \xi\right) d \xi\right|<M \Lambda_{n} \int_{0}^{1 / \Delta_{n}}\left|\Psi_{1}\left(\xi_{0}, \xi\right)\right| d \xi=M \Phi_{1}\left(\xi_{0}, 1 / \Lambda_{n}\right)<M \eta .
$$

Moreover, an application of the second of formulas (98) followed by an integration by parts yields, when $n$ is sufficiently large and $\sigma_{1}>0$, the inequality

$$
\begin{aligned}
& \left|\int_{1 / \Lambda_{n}}^{e} I(\xi, n) \Psi\left(\xi_{0}, \xi\right) d \xi\right|<M \Lambda_{n}{ }^{-\sigma_{1}} \int_{1 / \Lambda_{n}}^{e} \frac{\left|\Psi_{1}\left(\xi_{0}, \xi\right)\right|}{\xi^{\sigma_{1}+1}} d \xi \\
& =M \Lambda_{n}{ }^{-\sigma_{1}}\left\{\left.\frac{\Phi_{1}\left(\xi_{0}, \xi\right)}{\xi^{\sigma_{1}}}\right|_{1 / \Lambda_{n}} ^{e}+\left(\sigma_{1}+1\right) \int_{1 / \Lambda_{n}}^{e} \frac{\Phi_{1}\left(\xi_{0}, \xi\right)}{\xi^{\sigma_{1}+1}} d \xi\right\}, \\
& <M\left\{\frac{\Phi_{1}\left(\xi_{0}, \xi\right)}{\left(\Lambda_{n} \epsilon\right)^{\sigma_{1}}}+\Phi_{1}\left(\xi_{0}, 1 / \Lambda_{n}\right)+\left(\sigma_{1}+1\right) \eta \Lambda_{n}{ }^{-\sigma} \int_{1 / \Lambda_{n}}^{e} \frac{d \xi}{\xi^{\sigma_{1}+1}}\right\}<M_{1} \eta .
\end{aligned}
$$

* Lebesgue, Leçons sur les Séries Trigonométriques, Paris, 1905, p. 96.

† Hardy, Proceedings of the London Mathematical Society, (2), vol. 12 (1913), p. 368. 
It follows that when $\sigma_{1}$ is positive the second integral in (100) is of the order $o(1)$, and hence that at almost all points within the interval

$$
\mathcal{F}_{n}{ }^{0}(x) \cdot \rightarrow\left(\begin{array}{c}
\bar{f}_{1}(x) \\
0
\end{array}\right) \text {. }
$$

The discussion of $\mathscr{F}_{n}^{1}(x)$. is precisely similar to that of $\mathcal{F}_{n}^{0}(x)$. just completed. Since the argument of $\$ 16$ was based only on the integrability of the functions $f_{1}(x), f_{2}(x)$, the reasoning of that section requires no modification and we may summarize the results as follows.

THEOREM 2. If the conditions of Theorem 1 are satisfied, and if, furthermore, $\sigma_{i}>0, i=1,2$, then the expansion (60) is summable of order $\left(\sigma_{1}, \sigma_{2}\right)$ to the value $\mathcal{F}(x)$. at almost all points $x$ in the interior of the interval $(0,1)$.

CoRollary. If the conditions of Theorem 1, Corollary 2, are satisfied and if, furthermore, $\sigma_{i}>0$, then the expansion $I_{i n}\left(f_{1}, f_{2}\right), i=1,2$, is summable of order $\sigma_{i}$ to the value $f_{i}(x)$ at almost all points within the interval $(0,1)$.

18. The expansion when $\alpha=\pi / 2$, and $0<x<1$. When $\alpha=\pi / 2$, the rays (37) coincide with the axis of reals, and the constants $\nu_{i}$ of formula (6b) have the values $\nu_{1}=i, \nu_{2}=-i$. If it is observed that in this case

$$
D(\lambda) e^{\lambda i\left|R_{2}\right|}
$$

is similar in structure to $D(\lambda)$ in the case $\alpha=0$, with $\lambda$ replaced by $\lambda i$ and $R_{j}$ replaced by $\left|R_{j}\right|$, it becomes evident that the discussions of $\$ \$ 15,16$, and 17 are easily adapted to apply to the case in hand. The hypothesis to be made in correspondence to (86) is that

$$
\left.D_{1}(\lambda) \neq 0, \quad D_{2}(\lambda) \neq 0 \quad \text { (if } \alpha=\pi / 2\right),
$$

and by $\$ 13$ we are to choose $\mu=2$. The resulting theorems are the following.

Theorem 3. If $\alpha=\pi / 2$ and $D_{1}(\lambda) D_{2}(\lambda) \not \equiv 0$, let $\bar{\kappa}_{i}, i=1,2$, designate the largest of the numbers

$$
\begin{aligned}
& \pm\left(\kappa_{2}-\kappa_{1}\right), \quad \kappa_{3}-\kappa_{2}-\delta_{i 2}, \quad \kappa_{0}-\kappa_{1}-\delta_{i 2}, \quad \kappa_{4}-\kappa_{2}-\delta_{i 2}, \quad \kappa_{4}-\kappa_{1}-\delta_{i 2}, \\
& \kappa_{0}-\kappa_{2}-\delta_{i 1}, \quad \kappa_{3}-\kappa_{1}-\delta_{i 1}, \quad \kappa_{5}-\kappa_{2}-\delta_{i 1}, \quad \kappa_{5}-\kappa_{1}-\delta_{i 1} .
\end{aligned}
$$

Then with $\mu=2$ and $\sigma_{i} \geqq \bar{\kappa}_{i}$ the expansion (60) is summable of order $\left(\sigma_{1}, \sigma_{2}\right)$ to the value $(96)$ at every point $x$ within the interval $(0,1)$ in some neighborhood of which $\mathfrak{F}(x) \cdot$ is of bounded variation.

THEOREM 4. If the conditions of Theorem 3 are satisfied and if, furthermore, $\sigma_{i}>0, i=1,2$, then the expansion is summable of order $\left(\sigma_{1}, \sigma_{2}\right)$ to the value $\mathcal{F}(x) \cdot$ at almost all points within the interval $(0,1)$. 
It is clear that corollaries similar to those of Theorems 1 and 2, may also be appended respectively to the Theorems 3 and 4 .

19. The expansion when $0<\alpha<\pi / 2$, and $0<x<1$. The details of the discussion of the integrals (85) differ somewhat when $0<\alpha<\pi / 2$ from those of the preceding sections. The hypothesis to be made for this case is that no term of the characteristic equation (35) is lacking, i.e.,

$$
\left.D_{j}(\lambda) \not \equiv 0, \quad j=0,1,2,3 \quad \text { (if } 0<\alpha<\pi / 2\right) .
$$

Moreover, as observed in $\$ 13$, we shall, in the case of summability as distinguished from that of convergence of the expansion, restrict the consideration by the further hypothesis that $\alpha$ is commensurable with $\pi$.

The rays (37) together with the axes of reals and imaginaries divide the contour $C_{n}$ into eight arcs which we designate by $C_{n}^{(j, i)}$, where $C_{n}^{(j, 1)}$ and $C_{n}^{(j, 2)}$ are the arcs on the $j$ th quadrant taken in counter-clockwise succession. Since $R_{j}(x)=\nu_{j}\left|R_{j}(x)\right|$, whereas, on the arc $C_{n}^{(1,1)}$, we have $\operatorname{Re}\left(\lambda \nu_{1}\right) \geqq 0$, $\operatorname{Re}\left(\lambda \nu_{2}\right)>0$, we may write

$$
D^{-1}(\lambda)=D_{3}^{-1}(\lambda) e^{-\lambda \nu_{1}\left|R_{1}\right|-\lambda \nu_{2}\left|R_{2}\right|} O(1), \quad \text { on } \operatorname{arc} C_{n}^{(1,1)} .
$$

On the $\operatorname{arc} C_{n}^{(1,2)}$ we have, on the other hand, $\operatorname{Re}\left(\lambda \nu_{1}\right) \leqq 0, \operatorname{Re}\left(\lambda \nu_{2}\right)>0$, and we write accordingly

$$
D^{-1}(\lambda)=D_{2}^{-1}(\lambda) e^{-\lambda \nu_{2}\left|R_{2}\right|} O(1), \quad \text { on } \operatorname{arc} C_{n}{ }^{(1,2)} .
$$

With these values substituted respectively into the formulas (87), we obtain the relations

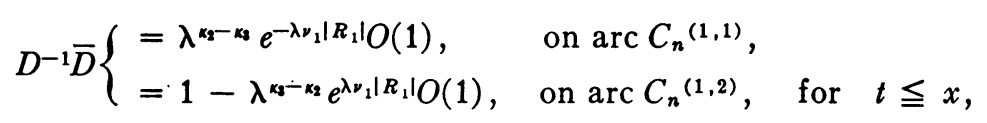

the values to be diminished by one for $t>x$.

On the portion of the contour $C_{n}$ in the second quadrant, we use the second of equations (87) together with the evaluations

$$
D^{-1}(\lambda)=D_{0}^{-1}(\lambda) O(1), \quad \text { when } \quad D_{1}(\lambda) \geqq D_{3}(\lambda) e^{\lambda \nu_{2}\left|R_{2}\right|},
$$

and

$$
D^{-1}(\lambda)=D_{2}^{-1}(\lambda) e^{-\lambda \nu_{2}\left|R_{2}\right|} O(1), \text { when } \quad D_{1}(\lambda)<D_{3}(\lambda) e^{\lambda \nu_{2}\left|R_{2}\right|},
$$

and obtain in this way the formula

(101) $D^{-1} \bar{D}=1-\lambda^{h_{0}} e^{\lambda_{\nu_{1}}\left|R_{1}\right|} O(1)$, on $\operatorname{arcs} C_{n}{ }^{(2,1)}$ and $C_{n}{ }^{(2,2)}$, for $t \leqq x$.

Here $h_{0}$ designates the larger of the numbers $\kappa_{3}-\kappa_{2}$ and $\kappa_{1}-\kappa_{0}$. In similar fashion we find also the further formulas 


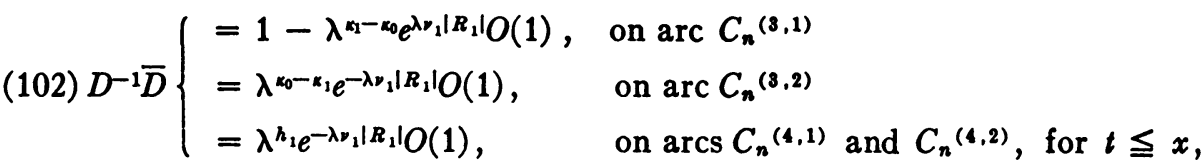

with $h_{1}$ defined as the larger of the numbers $\kappa_{0}-\kappa_{1}$ and $\kappa_{2}-\kappa_{3}$. In both formulas (101) and (102) the values given are to be diminished by one when $t>x$.

The integral (85) with $l=0$ may now be written explicitly, and is found to be given by the formula

$$
\begin{aligned}
& \mathcal{F}_{n}^{0}(x) \cdot=\frac{-1}{2 \pi i}\left(\int_{0}^{x} \int_{C_{n^{\prime}}} s_{i n} \lambda^{-\delta_{i 2} \omega_{i, 11}}(x, t, \lambda) e^{\lambda \nu_{1}\left|R_{1}(x, t)\right|} d \lambda d t\right. \\
& -\int_{x}^{1} \int_{C_{n^{\prime \prime}}} s_{i n} \lambda^{-\delta_{i 2} \omega_{i, 11}}(x, t, \lambda) e^{\lambda \nu_{1}\left|R_{1}(x, t)\right|} d \lambda d t \\
& +\int_{0}^{1} \int_{C_{n^{\prime}}{ }^{\prime}} s_{i n} \lambda^{h-\delta_{i 3}} \omega_{i, 11} e^{\lambda \nu_{1}\left|R_{1}(x)\right|+\lambda \nu_{1}\left|R_{1}(1, t)\right|} O(1) d \lambda d t \\
& \left.+\int_{0}^{1} \int_{C_{n}{ }^{\prime \prime}} s_{i n} \lambda^{h-\delta_{i 3} 2} \omega_{i, 11} e^{-\lambda \nu_{1}\left|R_{1}(1, x)\right|-\lambda \nu_{1} \mid R_{1}(t)} O(1) d \lambda d t\right) \text {, }
\end{aligned}
$$

$h$ being the largest of the numbers $\pm\left(\kappa_{3}-\kappa_{2}\right)$ and $\pm\left(\kappa_{1}-\kappa_{0}\right)$, while $C_{n}^{\prime}$ and $C_{n}{ }^{\prime \prime}$ represent respectively the portions of $C_{n}$ on which $\operatorname{Re}\left(\lambda \nu_{1}\right) \leqq 0$ and on which $\operatorname{Re}\left(\lambda \nu_{1}\right)>0$. We observe now that this formula (103) differs from (90) only in that $\lambda$ is replaced by $\lambda \nu_{1}$ and $R_{i}$ by $\left|R_{i}\right|$. Hence the discussion given subsequent to formula (90) may be repeated with slight modifications as applied to the formula (103) and as conclusion we obtain again the relation (94), provided in this case that $\sigma_{i} \geqq \pm\left(\kappa_{3}-\kappa_{2}\right)-\delta_{i 2}, \pm\left(\kappa_{1}-\kappa_{0}\right)-\delta_{i 2}$.

In precisely similar fashion the result (95) may now be derived under the condition that $\sigma_{i} \geqq \pm\left(\kappa_{2}-\kappa_{0}\right)-\delta_{i 1}, \pm\left(\kappa_{3}-\kappa_{1}\right)-\delta_{i 1}$, and the conclusion that $\mathcal{F}_{n}^{2}(x)$. and $\mathcal{F}_{n}^{8}(x)$. each approach $\mathcal{O}$ as a limit follows if $\sigma_{i} \geqq \kappa_{4}-\kappa_{j}-\delta_{i 2}$, $\kappa_{5}-\kappa_{j}-\delta_{i 1}, j=0,1,2,3$. The results, then, may be summarized as follows.

Theorem 5. If $0<\alpha<\pi / 2$ and $D_{j}(\lambda) \neq \equiv, j=0,1,2,3$, let $\bar{\kappa}_{i}, i=1,2$, designate the largest of the numbers

$$
\begin{array}{lll} 
\pm\left(\kappa_{3}-\kappa_{2}\right)-\delta_{i 2}, & \pm\left(\kappa_{1}-\kappa_{0}\right)-\delta_{i 2}, & \kappa_{4}-\kappa_{j}-\delta_{i 2}, \\
\pm\left(\kappa_{2}-\kappa_{0}\right)-\delta_{i 1}, & \pm\left(\kappa_{3}-\kappa_{1}\right)-\delta_{i 1}, & \kappa_{5}-\kappa_{j}-\delta_{i 1}, \quad j=0,1,2,3 .
\end{array}
$$

Then (i) if $\bar{\kappa}_{i}=0$ the expansion converges to the value (96) at every point in the interior of the interval $(0,1)$ in some neighborhood of which $\mathcal{F}(x)$. is of bounded variation; (ii) if $\bar{\kappa}>0$, and furthermore $\alpha / \pi$ is rational, then with $\sigma_{i} \geqq \bar{\kappa}_{i}$ the expansion is summable of order $\left(\sigma_{1}, \sigma_{2}\right)$ to the value (96) at every inner point of $(0,1)$ in some neighborhood of which $\mathcal{F}(x) \cdot$ is of bounded variation. 
THEOREM 6. If the conditions of Theorem 5 are satisfied and $\sigma_{i}>0$, the expansion is summable of order $\left(\sigma_{1}, \sigma_{2}\right)$ to the value $\mathcal{F}(x) \cdot$ at almost all points within the interval $(0,1)$.

The analogous facts for the expansions (72) may obviously be covered in corollaries to these theorems as was done in the case of Theorems 1 and 2.

20. The expansion at $x=1$ when $\alpha=0$. The argument from which the convergence of the integrals in the preceding sections was concluded depends explicitly upon the fact that $x$ is an interior point of the interval $(0,1)$. Not only must the reasoning be elaborated when $x$ is an end point of the interval, but we shall be able to conclude summability of the expansion only in the case of functions $f_{1}(x)$, and $f_{2}(x)$, satisfying suitable restrictions. For the sake of brevity we shall confine our considerations to the case $x=1$ and $\alpha=0$. The discussions required for the case $x=0$, and for other values of $\alpha$ may be given in an entirely analogous manner. To simplify the statement of the results we shall consider $\mathcal{F}(x) \cdot$ defined at $x=0$ and $x=1$ so that $\mathcal{F}(0) \cdot=\mathcal{F}(0+) \cdot$, and $\mathfrak{F}(1) \cdot=\mathfrak{F}(1-) \cdot$ whenever these limits exist.

We suppose, then, that the conditions of Theorem 1 are fulfilled. Then a review of the integrals in the expressions for $\mathcal{F}_{n}^{l}(x) \cdot$ in $\$ \$ 15$ and 16 reveals the fact that the integrals over the arc $C_{n}^{\prime}$ are of the same character for $x=1$ as for $0<x<1$, and hence that they converge to the value $\frac{1}{2} \bar{F}(1) \cdot$ But from formula (58) this value is zero, and hence we need consider these integrals no further. The integrals over the arc $C_{n}^{\prime \prime}$, however, do require further consideration. Thus the final integral in formula (90) was evaluated by means of Lemma IV, while the applicability of that lemma depends upon the fact that $\left\{R_{1}(1, x)+R_{1}(t)\right\}>0$, a condition which is not fulfilled when $x=1$. The discussion of the integral by other means requires a more precise expression for the integrand. To obtain this we shall make use of the identity

$$
\frac{1}{D(\lambda)}=\frac{1}{D_{3}(\lambda) e^{\lambda R_{1}+\lambda R_{2}}}\left\{-\frac{D_{0}+D_{1} e^{\lambda R_{1}}+D_{2} e^{\lambda R_{2}}}{D}+1\right\} \text {. }
$$

Consider the fraction within the brace of this expression. It is obviously bounded. We may, however, also obtain an evaluation of it by utilizing the first of formulas (88), and hence (104) may be written in the form

$$
D^{-1}(\lambda)=\lambda^{-\kappa_{3}} e^{-\lambda R_{1}-\lambda R_{3}} N(\lambda)+D_{3}^{-1} e^{-\lambda R_{1}-\lambda R_{2}},
$$

in which $N(\lambda)$ is subject to the alternative evaluations

$$
N(\lambda)\left\{\begin{array}{l}
=O(1), \\
=\lambda^{\alpha_{0}-\alpha_{3}} e^{-\lambda R_{1}-\lambda R_{2}} O(1)+\lambda^{\alpha_{1}-\alpha_{2}} e^{-\lambda R_{2}} O(1)+\lambda^{\alpha_{2}-\alpha_{3}} e^{-\lambda R_{1}} O(1) .
\end{array}\right.
$$


We return now to the in tegrals over arc $C_{n}^{\prime \prime}$ in formula (90). When $x=1$ the first of these integrals clearly does not appear. On the other hand, we obtain in place of the second integral when the first of formulas (88) and the formula (105) are substituted in the respective terms of $D^{-1} \bar{D}$ the three integrals

$$
\begin{aligned}
& \int_{0}^{1} \int_{C_{n}^{\prime \prime}} s_{i n} \lambda^{\alpha_{0}-\alpha_{3}-\delta_{i 2}} e^{-\lambda R_{2}-\lambda R_{1}(t)} O(1) \omega_{i, 11}(1, t, \lambda) d \lambda d t, \\
& \int_{0}^{1} \int_{C_{n^{\prime \prime}}{ }_{i n} \lambda^{\alpha_{2}-\alpha_{3}-\delta_{i 2}} e^{-\lambda R_{1}(t)}\left[\alpha_{2}\right] N(\lambda) \omega_{i, 11}(1, t, \lambda) d \lambda d t,} \int_{0}^{1} \int_{C_{n^{\prime \prime}}{ }^{\prime \prime} \lambda^{\alpha_{2}-\alpha_{3}-\delta_{i 3}} e^{-\lambda R_{1}(t)}\left[\frac{\alpha_{2}}{\alpha_{3}}\right] \omega_{i, 11}(1, t, \lambda) d \lambda d t .}
\end{aligned}
$$

The first of these, i.e., (107), is found directly to be of order $o(1)$ by the arguments of $\S 15$. The second, however, i.e., (108), requires a more careful analysis. We shall discuss it in detail only in so far as the integration over the part of $C_{n}^{\prime \prime}$ which lies in the first quadrant is concerned. No essential modification of the argument is necessary to adapt it to the discussion of the integration over the remaining part of $C_{n}^{\prime \prime}$. To begin with we set

$$
\lambda=i \Lambda_{n} e^{-i x} .
$$

Then the arc of $C_{n}^{\prime \prime}$ on the first quadrant is given by $0 \leqq \chi \leqq \pi / 2$, and this we divide by a point $\lambda_{a}$ into arc (1) and arc (2) as follows:

where

$$
\begin{array}{ll}
\operatorname{arc}(1): & 0 \leqq \chi \leqq \chi_{a}, \\
\operatorname{arc}(2): & \chi_{a}<\chi \leqq \pi / 2,
\end{array}
$$

$$
\chi_{a}=\frac{A \log \Lambda_{n}}{\Lambda_{n}} \text {. }
$$

In this we tentatively specify $A$ merely as a positive constant.

On the arc (1) now we use for $N(\lambda)$ the first of the evaluations (106). By Lemma II it follows then that the integration over this arc contributes to (108) a quaditity which is of the order

$$
\Lambda_{n}{ }^{\kappa_{2}-\kappa_{3}-\delta_{i 2}}\left\{\frac{A \log \Lambda_{n}}{\Lambda_{n}}\right\}^{\sigma_{i}} O(1),
$$

i.e., of the order $o(1)$ for any choice of the constant $A$ provided $\sigma_{i}>\kappa_{2}-\kappa_{3}-\delta_{i 2}$. Since the condition $\sigma_{i} \geqq \bar{\kappa}_{i}$ imposed in Theorem 1 admits also of the possibility $\sigma_{i}=\kappa_{2}-\kappa_{3}-\delta_{i 2}$, we must also consider this case. However, the argument requires modification only in the case of the integral arising from the first term of $\omega_{i, 11}(1, t, \lambda)$, and we may conclude this integral also to be of the order 
$o(1)$ by the use of the Lebesgue theorem B if we suppose the function $f_{1}(t)$ to be of bounded variation in some neighborhood of $t=0$.

We turn next to the contribution to (108) by the integration over the arc (2). To begin with we observe that on this arc

$$
\left|\lambda^{A R_{i}} e^{-\lambda R_{i}}\right|=\left|e^{\left(\lambda_{a}-\lambda\right) R_{i}}\right| \cdot\left|\lambda^{A R_{i}} e^{-\lambda_{a} R_{j}}\right|,
$$

while in this expression the first factor on the right is bounded since $\operatorname{Re}\left(\lambda_{a}-\lambda\right) \leqq 0$. The second factor, on the other hand, may be written in the form

$$
e^{\left.\Lambda_{n} R_{j} \mid x_{a}-\sin \chi_{a}\right\}}
$$

from which it is seen to approach unity as $\Lambda_{n} \rightarrow \infty$. It follows, therefore, in virtue of the second of formulas (106), that if $A$ is chosen sufficiently large we have on $\operatorname{arc}(2)$

$$
\lambda^{\alpha_{2}-\alpha_{0}-\delta_{i 2}} e^{-\lambda R_{1}(t)}\left[\alpha_{2}\right] N(t)=\lambda^{-1} O(1) .
$$

By Lemma I then the integration over this arc contributes to (108) a quantity which is of the order $o(1)$, and in consequence the entire integral (108) is of that order.

The integral (109) remains to be evaluated to complete the discussion of $\mathcal{F}_{n}^{0}(1)$. For this evaluation and that of the corresponding integrals in the expressions $\mathcal{F}_{n}{ }^{l}(1) \cdot, l=1,2,3$, we shall impose upon the functions $f_{1}(t)$ and $f_{2}(t)$ the restrictions that the limits

$$
\lim _{t \rightarrow 0} \frac{\bar{f}_{1}(t)}{t^{\tau_{i 1}}}, \quad \text { and } \quad \lim _{t \rightarrow 0} \frac{\bar{f}_{2}(t)}{t^{\tau_{i 2}}} \text {, exist for } i=1,2,
$$

where $\tau_{i j}$ designates the largest of the numbers $\kappa_{2}-\kappa_{3}-\delta_{i 2}-\delta_{j 2}, \kappa_{1}-\kappa_{3}-\delta_{i 1}-\delta_{j 1}, \kappa_{4}-\kappa_{3}-\delta_{i 2}-\delta_{j 1}, \kappa_{5}-\kappa_{3}-\delta_{i 1}-\delta_{j 2}$.

A review of the hypothesis of Theorem 1 shows that the numbers $\tau_{i j}$ do not exceed the value of $\bar{\kappa}_{i}$ and hence that any $\sigma_{i}$ chosen in accordance with Theorem 1 satisfies the relation $\sigma_{i} \geqq \tau_{i j}$. We shall show that if the hypothesis (110) is satisfied then the expansion is summable at $x=1$.

Consider then the integral (109). Using formula (77) we may write

$$
\begin{aligned}
\lambda^{\kappa_{2}-\alpha_{3}-\delta_{i 2}}\left[\frac{\alpha_{2}}{\alpha_{3}}\right] \omega_{i, 11}(1, t, \lambda) & \\
=\bar{f}_{1}(t) \sum_{m=0}^{\kappa_{2}-\kappa_{3}-\delta_{i 2}} \lambda^{m} \beta_{i m}^{(1)}(t)+\bar{f}_{2}(t) & \sum_{m=0}^{\alpha_{2}-\alpha_{3}-\delta_{i 2}-1} \lambda^{m} \beta_{i m}^{(2)}(t) \\
& +\frac{E(t, \lambda) \bar{f}_{1}(t)+E(t, \lambda) \bar{f}_{2}(t)}{\lambda}
\end{aligned}
$$


the coefficients $\beta_{i j}^{(k)}(t)$ as well as the functions $E(t, \lambda)$ being integrable on the interval $(0,1)$. The integrals arising in (1.09) from the final terms on the right of (111) are of order $o(1)$ by Lemma $I$. Of the integrals due to the remaining terms in (111) we consider next that arising from the general term involving $\bar{f}_{1}(t)$, i.e.,

$$
\int_{0}^{1} \int_{C_{n^{\prime \prime}}} s_{i n}(\lambda) \lambda^{m} e^{-\lambda R_{1}(t)} \beta_{i m}^{(1)}(t) \bar{f}_{1}(t) d \lambda d t .
$$

For any integer $l$ not exceeding $\sigma_{i}$ we readily verify that

$$
\frac{d^{l}}{d \lambda^{l}}\left\{s_{i n}(\lambda) \lambda^{m}\right\}=\left\{1-\left(\frac{\lambda}{\Lambda_{n}}\right)^{\mu}\right\}^{\sigma_{i-l}} \lambda^{m-l} P\left(\frac{\lambda^{\mu}}{\Lambda^{\mu}}\right)
$$

where $P$ is used to designate a polynomial. Since the right-hand member of this equation vanishes at the ends of the $\operatorname{arc} C_{n}{ }^{\prime}$ when $\sigma_{i}>l$, we obtain for (112) by an integration by parts $\tau_{i 1}$ times repeated the form

$$
\int_{0}^{1} \int_{C_{n^{\prime \prime}}}\left\{1-\left(\frac{\lambda}{\Lambda_{n}}\right)^{\mu}\right\}^{\sigma_{i}-r_{i 1}} \lambda^{m-\tau_{i 1} e^{-\lambda R_{1}(t)}} P\left(\frac{\lambda^{\mu}}{\Lambda_{n}}\right) \beta_{i m}^{(1)}(t) \frac{\bar{f}_{1}(t)}{R_{1}(t)^{r_{i 1}}} d \lambda d t
$$

Inasmuch as $r_{1}(t)$ is positive everywhere on $(0,1)$ the integrand of $(113)$ is integrable with respect to $t$ by virtue of hypothesis (110). Hence by Lemma I all the integrals (113) for which $m<\tau_{i 1}$ are of the order $o$ (1). If $\kappa_{2}-\kappa_{3}-\delta_{i 2}<\tau_{i 1}$, this accounts for all the integrals due to the first sum in (111). However, if $\kappa_{2}-\kappa_{3}-\delta_{i 2}=\tau_{i 1}$, the integral (113) for which $m=\tau_{i 1}$ remains to be considered. A simple extension of the Lemmas III and V to account for the factor $P\left(\lambda^{\mu} / \Lambda_{n}^{\mu}\right)$ in the integrand suffices for this consideration, and we find that the integral converges to a value

$$
C_{i 1}^{(0)} \lim _{t \rightarrow 0}\left\{\frac{\bar{f}_{1}(t)}{t^{r_{i 1}}}\right\},
$$

if $\sigma_{i}>\tau_{i 1}$, or in the case $\sigma_{i}=\tau_{i 1}$, provided the function $\bar{f}_{1}(t) / t^{\tau_{i 1}}$ is of bounded variation in some neighborhood of $t=0$. In this result $C_{i 1}^{(0)}$ is a constant determined by the differential system and is independent of the functions $f_{i}(t)$.

A similar discussion leads to the evaluation of the integrals arising from the terms of the second sum in (111) and we obtain in this way the result that under the hypotheses made

$$
\mathcal{F}_{n}{ }^{0}(1) \cdot \rightarrow\left(C_{i 1}^{(0)} \lim _{t \rightarrow 0}\left\{\frac{\bar{f}_{1}(t)}{t^{\tau}{ }^{i 1}}\right\}+C_{i 2}^{(0)} \lim _{t \rightarrow 0}\left\{\frac{\bar{f}_{2}(t)}{t^{\tau_{i 2}}}\right\}\right)=\lim _{t \rightarrow 0}\left(\frac{C_{i j}^{(0)}}{t^{\tau_{i j}}}\right) \overline{\mathscr{F}}(t) .
$$


The discussions of the integrals in the expressions $\mathcal{F}_{n}^{l}(1)$. for $l=1,2,3$ follow on similar lines and we obtain thus as a total result, since by formula (59), $A(1) \cdot=\mathcal{F}(1) \cdot$, the result

$$
, \mathcal{F}_{n}(1 \mathfrak{F} \cdot) \rightarrow \mathfrak{F}(1) \cdot+\lim _{t \rightarrow 0}\left(\frac{C_{i j}}{t^{\tau_{i j}}}\right) \overline{\mathcal{F}}(t) .
$$

We see, therefore, that the expansion is summable at $x=1$ if the components of the vector $\bar{F}(t)$. vanish to a sufficiently high degree at $t=0$. In quite the same manner we should find that the expansion is summable at $x=0$ if the components of the given vector vanish to a prescribed degree at $x=1$.

A special case of importance is that in which the numbers $\tau_{i j}$ are all zero, for in this case the final term of (114) is a constant matrix multiplied by $\mathcal{F}(0)$. and this is $\mathcal{O}$ by formula (58). Hence the expansion converges then at $x=1$ to the value $F(1) \cdot$. If in accordance with Theorem 1 the expansion actually converges, then since $\sigma_{i} \geqq \tau_{i j}$ it follows that the constants $\tau_{i j}$ are zero and, moreover, that the same is true of the corresponding exponents associated with the discussion of the expansion at $x=0$. Hence the expansion then converges also at both end points to the respective values of $\mathcal{F}(x)$.

The discussion in the case $\alpha=\pi / 2$ is similar to that given in detail above, and the results differ only in that the expansion is summable at $x=1$ if the components of the vector $\bar{F}(t)$. vanish to a prescribed degree at $t=1$, while summability at $x=0$ is assured by the vanishing of $\bar{F}(t)$ at $t=0$. In the case $0<\alpha<\pi / 2$ a combination of the phenomena described occurs and the proof of summability at either end point is found to be dependent upon the suitable vanishing of the vector $\bar{F}(t)$. at both end points of the interval. We summarize these observations roughly as follows:

THEOREM 7. If the expansion under the conditions of the respective theorem above is convergent (as distinguished from summable) at the interior points of the interval, then it is also convergent to the value of the given vector at the end points. If the expansion is summable but not convergent at the inner points, then it is summable at the end points also provided the vector $\bar{F}(t)$. vanishes at $t=0$ and $t=1$ to certain degrees prescribed by the differential system.

UNIVERSITY OF WISCONSIN, Madison, Wrs. 